\title{
Cortical white matter: beyond the pale remarks, main conclusions and discussion
}

\section{Javier DeFelipe ${ }^{*}$, R. Douglas Fields ${ }^{2}$, Patrick R. Hof ${ }^{3}$, Malin Höistad ${ }^{4}$, Ivica Kostovic 5 , Gundela Meyer ${ }^{6}$ and Kathleen S. Rockland ${ }^{7 * *}$}

\author{
- Laboratorio de Circuitos Corticales, Centro de Tecnología Biomédica, Universidad Politécnica de Madrid and Instituto Cajal (CSIC), Madrid, Spain \\ 2 National Institute of Child Health and Human Development, National Institutes of Health, Bethesda, MD, USA \\ 3 Department of Neuroscience, Mount Sinai School of Medicine, New York, NY, USA \\ 4 Mount Sinai School of Medicine, New York, NY, USA \\ 5 University of Zagreb, Zagreb, Croatia \\ 6 Departamento de Anatomía, Facultad de Medicina, Universidad de La Laguna, Tenerife, Spain \\ 7 RIKEN-MIT Center for Neural Circuit Genetics, MIT, Cambridge, MA 02139 \\ Correspondence: *defelipe@cajal.csic.es; **kathrock@mit.edu
}

\section{INTRODUCTION}

This Special Topic "Cortical white matter: beyond the pale" includes 10 articles from 32 authors. These articles in this offer a summary of some of the current thinking regarding myelin, and the associated cellular populations in the white matter. The articles range from the micro-level of ultrastructure and molecular factors, to populational organization, and cognitive effects. All the articles devote at least some discussion to myelin in psychiatric conditions, raising the prospect of new paradigms of investigation and treatment. In this article the main conclusions, and some of what the host editors (Kathleen Rockland and Javier DeFelipe) consider the most interesting remarks, have been extracted from each of the individual articles. These commentaries are not necessarily directly derived from the original work of the authors, and may be the result of the collective work of several different laboratories. This is followed by a section dedicated to more general comments and a discussion of the issues raised. The authors who have participated in this article are listed in alphabetical order.

\section{A commentary on}

Neurons in the white matter of the adult human neocortex by SuarezSola, M., Gonzalez-Delgado, F. J., Pueyo-Morlans, M., Medina-Bolivar, O. C., Hernandez-Acosta, N. C., Gonzalez-Gomez, M., and Meyer, G. (2009). Front. Neuroanat. 3:7. doi: 10.3389/neuro.05.007.2009.

\section{REMARKS AND MAIN CONCLUSIONS}

1. In the human brain, the white matter (WM) underlying the cerebral neocortex is highly developed and occupies a much larger volume than in other mammals. Although the dominant components of the WM are the complex fiber tracts, their ensheathing myelin and supporting glia, there are also large numbers of neurons dispersed among the fibers, termed the "interstitial neurons" (IN). They are prominent in the primate WM, and poorly developed in the rodent. The species differences may reflect a direct correlation between the size of the cortical gray matter, the amount of WM connecting the neocortex, and the number of IN.

2. In human, the border between gray and WM is relatively sharply defined at the bottom of the sulci and along the flanks of the gyri, but more difficult to delimit at the crowns or apices of the gyri, where radial fiber fascicles intermingle with radial rows of layer VIb neurons and IN seem to be continuous with neurons of layer VIb.

3. The highest density of IN is in the WM immediately subjacent to the gray matter, in the zone that contains the association or " $U$ " fibers of the cortical convolutions, and then gradually decreases with increasing distance from the gray matter. Very few neurons lie among the long fiber tracts in the deep WM, such as internal capsule, superior and inferior longitudinal fasciculi, or corpus callosum. However, there is no sharp boundary between the superficial WM, rich in IN, and the deep WM, where IN are sparse. There may also be regional differences in the density of IN, with lowest numbers in the visual cortex, and higher numbers in the frontal and prefrontal cortex.

4. The IN display a variety of morphologies ranging from pyramidal-like to bipolar and multipolar. They can be classified into the two main neuronal categories also present in the gray matter, namely excitatory glutamatergic cells and inhibitory GABAergic neurons.

5. IN of the cortical WM are often referred to as "subplate" cells. During development, the subplate is a transient cell compartment just below the future layers VI-II, or "cortical plate". Birthdating studies in rodents and carnivores revealed that subplate neurons are generated at the same time as CajalRetzius cells in the marginal zone (or future layer I), and prior to the birth of cortical plate neurons.

6. Subplate cells perform multiple developmental functions: they extend pioneer fibers into the internal capsule and direct thalamo-cortical pathfinding, serve as transient synaptic targets for thalamocortical fibers, and provide a substantial glutamatergic input into the maturing cortical plate, helping in the establishment of ocular dominance columns in the primary visual cortex. As the cortical plate matures, many subplate neurons degenerate and undergo programmed cell death. The survivors continue into adult life as IN of the WM.

7. Subplate neurons are morphologically and neurochemically heterogeneous. The GABAergic subpopulations may express a variety of peptides such as neuropepide Y, somatostatin and/or cholecystokinin, or contain nitric oxide synthase. It 
is not known if developmental cell death affects specific cell classes within the subplate, or whether all subpopulations are equally reduced.

8. Although human subplate neurons are heterogeneous, a useful marker of the glutamatergic component is the putative transcription factor T-brain-1 (Tbr1). The chronology of Tbr1 expression in human fetuses can be traced to the early cortical plate at $10 \mathrm{GW}$, which is strongly Tbr1+. From 14 to $25 \mathrm{GW}$, large numbers of Tbr1+ neurons are continuously added to the subplate compartment, which increases in width concurrent with the growth of the cortical plate, although the highest density is always at the border between cortical plate and subplate. In perinatal brains, Tbr1-immunoreactivity changes from a nuclear to a cytoplasmic staining that is widely expressed in neurons in the cortical gray and white matter, and thus no longer useful as a marker molecule of the subplate. In the absence of molecules specific for the human subplate it is difficult to ascertain how many subplate cells survive as IN.

9. Altogether, these data show that the IN of the human WM are not identical to the early-born subplate neurons described in rodents and cat. Rather, the cell populations in the maturing WM seem to be complemented by newly arriving neurons generated at much later stages of corticogenesis. A possible explanation for the discrepancy across species may be the extraordinary increase in cortical connectivity during evolution, which leads to an increase in size and complexity of the WM in the primate brain. In parallel to the increase of the WM compartment, a continuous supply of IN may be required during the whole period of corticogenesis. This implies that primate IN are not just incidental remnants of early-born neurons, but rather seem to belong to a distinct neuronal system that is intimately connected to the WM and may carry out activities pertinent to this location.

10. The GABAergic interneurons of the cortical gray matter are highly diverse, and many attempts have been undertaken to classify them according to a variety of morphological and neurochemical properties. A recent inventory of mouse cortical interneurons has led to the identification of 13 cell classes based on the combined expression of the calcium-binding proteins calbindin $(\mathrm{CB})$, calretinin $(\mathrm{CR})$ and parvalbumin (PV), and neuropetides, such as vasointestinal polypeptide, NPY, cholecystokinin, somatostatin and choline acetyltransferase. Of the three calcium-binding proteins present in the cortical gray matter, only CB and CR are expressed in IN. PV+ cells are the largest group of cortical interneurons which includes basket cells and chandelier cells. PV is not found in the adult human $\mathrm{WM}$, and the few deep PV+ neurons occasionally found below the gray matter are more likely to represent displaced layer VI neurons. $\mathrm{CB}+\mathrm{IN}$ have been reported in the WM; they are concentrated in the superficial WM, often aligned along the gray/ WM border and most numerous in the apex regions.

11. CR is abundant in gray-matter interneurons mostly of supragranular layers, but its presence in the WM has not attracted much attention. This is surprising insofar as CR+ IN are the most prominent cell population in the superficial and deep adult human WM.
12. The distribution and relative prominence of interneurons expressing calcium-binding proteins are species and areadependent. $\mathrm{CR}+$ cells seem to be more prominent in primate than in rodent cortex, not only in the gray matter but also in the WM. In the mouse, CR+ interneurons derive from the caudal ganglionic eminence and migrate tangentially all over the cortex. By contrast, primate interneurons have a double origin, with early-born cells migrating from ganglionic eminences, and later-born cells deriving from the subventricular zone (SVZ) of the cortical wall. In particular, CR+ cells are very prominent in the SVZ and deep WM during late human fetal development.

13. Nitric oxide (NO) is a gaseous messenger molecule synthetized by several isoforms of the enzyme nitric oxide synthase (NOS). In the brain, two NOS forms are constitutively expressed, nNOS in neurons, and eNOS in endothelial cells. Nitrergic, i.e. NO-producing neurons, can be visualized by NADPH-diaphorase histochemistry, as well as by immunohistochemistry using anti-nNOS antibodies. There are two main cell classes: Type 1 NADPH-d neurons are intensely stained in a Golgi-like fashion, displaying medium size to large somata and long varicose processes. Most type 1 neurons are in the superficial WM, whereas type $2 \mathrm{NADPH}-\mathrm{d}$ neurons are restricted to the cortical gray matter. Type 2 neurons are only lightly stained and have small somata and short processes. Both types express GABA, and about $4 \%$ of type 1 neurons coexpress CB. Type 1 neurons can also express neuropeptide $\mathrm{Y}$ (NPY) and somatostatin. Although most GABAergic neurons are interneurons with local axons, some NADPH-d/nNOS+ neurons in the WM of rat, cat and monkey project over long distances to distant, functionally unrelated cortical areas.

14. One of the most interesting features of the type $1 \mathrm{NADPH}-\mathrm{d}$ neurons is their close association with blood vessels. Their axonal plexuses form a dense network around microvessels, and their long processes may contact distant arterioles and capillaries. Since NO is a potent vasodilator, NOS-containing neurons are thought to be involved in the coupling of metabolic changes related to neuronal function with local increases in blood flow. Due to their strategic location just below the cortical gray matter, NOS+ IN may be contacted by corticopetal fibers and, in response, act on neighboring microvessels. On the other hand, NPY is a powerful vasoconstrictor able to antagonize the vasodilating effect of NO that co-localizes with NOS in a subset of IN. Somatostatin and NPY are expressed in IN of the superficial WM. They act directly on smooth muscle cells of cortical arterioles, and may thus constrict cortical microvessels in an activity-dependent manner. The NOS/ NPY+ IN thus form part of the neural system involved in the coupling of cortical microvessels to neuronal activity.

15. The NOS+ IN in the cortical WM are an important component of the vasoactive pathways which also include subcortical cholinergic and serotoninergic systems. In fact, most NOSexpressing IN are cholinoceptive, meaning that they receive cholinergic fibers from the nucleus basalis. Since the axons of NOS+ IN may spread over considerable distances into the cortical gray matter, a single IN may coordinate local blood flow in neighboring and distant cortical areas in response to corticopetal and corticofugal activation. 
16. The subcortical WM and its resident IN have been associated with a variety of neurological and psychiatric disorders. Alterations of somatostatin, NPY and/or NADPH-d+ IN were observed in Alzheimer disease. However, schizophrenia is the disease which seems to show the most dramatic abnormalities of the WM. Diffusion tensor imaging revealed disturbances of myelin function and distribution, alterations of connectivity and integrity of fiber tracts such as the cingulate bundle and uncinate fasciculus. A higher incidence of changes occurrs in the frontal lobes, middle temporal structures including hippocampus and amygdala, and superior temporal gyrus, as well as in subcortical centers.

17. Schizophrenia also affects the IN in diverse ways. In the frontal lobe of schizophrenic patients, the IN density was decreased in the superficial WM, but increased in the deeper WM. NADPH-d+ IN show the same maldistribution as microtubule associated protein 2 (MAP2)+ cells in general. While some studies reported an increase in IN density in inferior parietal and dorsolateral prefrontal areas in deficit syndrome patients, others observed no change neither in superficial nor in deep WM. The conflicting reports on the changes of IN density in schizophrenia were summarized by Eastwood and Harrison, who observed a density increase in the superficial WM and no change in deeper compartments.

18. A special and rather minor subclass of IN expresses the extracellular matrix molecule Reelin, which is important for brain development and adult neuronal plasticity. In the adult cortical gray matter, Reelin is expressed by a subgroup of GABAergic interneurons; in the WM, very few scattered Reelin+ cells can be visualized using immunohistochemistry. Conversely, Reelin mRNA has been reported to be abundant in IN in the superior temporal cortex, and to be significantly reduced in schizophrenic patients, in keeping with the finding that alterations of Reelin expression are a putative vulnerability factor in schizophrenia and mood disorders.

19. Most discussions of IN changes in psychoses are based on the view that IN are remnants of the early-born subplate population. The early generation of IN in rodents and carnivores, and their maldistribution in schizophrenic patients, have led to the hypothesis that a migration defect of the subplate during embryonic or early fetal development underlies the pathogenesis of schizophrenia. As stated above, the developmental history of the subplate is very different in nonprimate mammals and in primates including human. An important task for future research would be a molecular taxonomy of all neuronal populations in the WM, similar to the work done on interneurons in the gray matter. It would be particularly important to differentiate between early-born components, probably related to transient roles of the subplate, and later-appearing resident cells, which may not be important for development but rather involved in activities proper to the adult WM. The recent discovery of subplate-specific molecules in mice is a useful step in this direction, and it is hoped that similar work will shed light on the origins, categories and functional roles of human IN.

\section{GENERAL COMMENTS AND DISCUSSION DeFELIPE}

Comment on points 4 and 13:

Are there studies that address the issue of synaptic connections of IN? studies on what is the proportion of these neurons that project over long distances?

\section{ROCKLAND}

Response to DeFelipe's comment:

Some articles in the next Special Topic (Cortical GABAergic neurons: stretching it) will talk about synaptic inputs, but I believe less is known about the output. I do not believe we have numbers yet about proportion. In part because there are multiple target structures, retrograde tracers are only partly useful in addressing this question.

\section{ROCKLAND}

Comment on points 2 and 5, and in general:

It may be worth remarking that "layer VIB" is used here to denote deep layer VI. This is not to be confused with "layer VIB" in rodents, sometimes also called "layer VII" and which constitutes a separate population. Questions of nomenclature are likely to be taken up in a subsequent Special Topic, as referenced above.

As these authors remark (points 6 and 7), the subplate neurons perform multiple functions developmentally, and themselves constitute a heterogeneous group. It is interesting to consider this in conjunction with Friedlander and Torres-Reveron, who emphasize the multiple and changing roles of WM neurons over a lifetime.

\section{HÖISTAD AND HOF}

Comment on interstital neurons, the nomenclature and species differences:

Suárez-Sola et al. discuss the so-called "interstitial neurons" (IN) of the WM of the adult human cortex, which are located directly subjacent to the cortical gray matter and are found in high numbers especially in the prefrontal cortex. Initially they were regarded as remnants from the subplate during neurodevelopment, however in primates, IN rather seem to belong to a distinct neuronal system that carries out activities pertinent to the subcortical WM.

Interestingly, some species have in adult stage large numbers of WM neurons, in particular large-brained mammals such as artiodactyls and cetaceans (Hof et al., 1999), while they are only rudimentary in the brain of non-primate mammals. As the authors indicate, such species differences may reflect a correlation between the size of the cortical gray matter, the amount of WM interconnecting the neocortex, and the number of IN. The higher number of IN in large-brained animals may possibly support one of the proposed functions of the IN, which is coordination of activity among neocortical regions.

These comparative differences may also possibly be indicative of species-specific importance, similar to other specialized neuron types (such as spindle "von Economo" neurons in the anterior cingulate and frontoinsular cortices, Betz cells in M1, or Meynert cells in V1). However, the IN are a very heterogenous population of neurons, some pyramidal-like and covered with spines, thus presumably glutamatergic, while others are multipolar or bipolar and express GABA and a variety of neuronal markers (such as 
$\mathrm{CB}, \mathrm{CR}$, neuropeptide $\mathrm{Y}$, somatostatin, and NOS). One of the proposed functions of the IN involves the coordinating of regulation of blood flow. Particularly in view of the presence of NO in some IN (as visualized by the presence of NADPH-d/nNOS+), and their close association with blood vessels, the IN have been suggested to be involved in the regulation of blood flow and neurovascular coupling. In addition, the expression of NPY, a powerful vasoconstrictor, could potentially antagonize the vasodilating effect of NO. Interestingly, comparable functions have been reported for select classes of neocortical interneurons characterized by their content of neuropeptides and particular morphologies (Cauli et al., 2004). If the IN have a role in blood flow regulation, it remains to be clarified however why are there fewer IN in visual cortex but higher numbers in frontal cortex, and also why they appear only rudimentary in smaller mammals such as rodents.

In the human, abnormalities of the IN in the frontal lobe have been observed in schizophrenia, although reports from different investigators are conflicting (summarized by Eastwood and Harrison, 2005). As the authors indicate, it is important to differentiate between early-born IN (probably related to transient roles of the subplate) and later-appearing resident cells, which may not be important for development but rather involved in activities proper to the adult WM.

\section{KOSTOVIC}

Authors and commentators discussed the significance of the prominence of subplate zone and neurons, and of the large numbers of interstitial neurons in the primate brain and particularly in human. The answer may be in the obvious fact that subplate prominence and number of interstitial neurons is related to the enormous increase of cortico-cortical connectivity in human (Kostovic and Rakic, 1990). The increase of complexity of cortico-cortical connections requires a prolonged existence of the subplate zone as well as prolonged developmental function of subplate neurons (Kostovic and Judas, 2006). This is also consistent with the increased number of gyri and gyral white matter in human and primates (remark 2).

In respond to Rockland comments about synaptic inputs, I also refer to the next special topic "Cortical GABAergic Projection Neurons" and in particular to the article "Subplate cells: amplifiers of neuronal activity in the developing cerebral cortex" of Luhmann, Kilb and Hanganu-Opatz. Without endogenous activity of the subplate neurons, cortex can not properly develop. Here we emphasize prolonged coexistence of subplate circuitry with the gradual formation of adult-like thalamo-cortical and cortico-cortical circuitry (Kostovic and Judas, 2006). Thus, the subplate zone should always be defined as a synaptic layer.

The glutamatergic component of subplate zone factor Tbr1 (remark 8) is recognized as a useful marker. There are several other specific markers found in the subplate zone, such as CplX3, CTGF, Nurr-1/Nr 4a2, Mox D1, CTGF and F-spondin (Ayoub and Kostovic, 2009).

Involvement of white matter neurons in neurological and psychiatric disorders (remarks 16 and 17) may have a developmental interpretation: selective vulnerability of the subplate zone and neurons during development. This may be a crucial factor in pathogenesis of several neurological mental and cognitive disorders (Kostovic and Judas, 2006).

\section{MEYER}

Response to the comments by Höistad and Hof regarding the number of interstitial neurons (IN) in different species. They argue that large brains have in general large amounts of cortical gray matter, accompanied by large numbers of cortical association fibers and thus an increased volume of cortical white matter, requiring large numbers of IN. The question is why the visual cortex has relatively few IN. I think that the connections of the primary visual cortex - Brodmann's area 17- are well defined and highly specific. On the one hand, area 17 itself is a narrow koniocortex, and the proportion of intracortically projecting neurons higher than in other cytoarchitectonic areas. On the other hand, callosal fibers are sparse if not absent in most of area 17, and they only increase toward the $17 / 18$ border, the representation of the vertical meridian. Area 17 is thus lacking a substantial component of the cortical white matter tracts, and in fact, macroscopic observation of the human striate area shows that the amount of white matter is smaller than in the nearby occipital association areas. By contrast, prefrontal areas have many and varied fiber connections which contribute to the huge volume of the underlying white matter. A possible additional function of IN may be that they serve as guideposts for distinct fiber fascicles, perhaps by establishing synaptic contacts. The primary visual cortex may thus not be the best model for studying IN, the more so since also during development subplate neurons below area 17 play important roles in specific visual functions, such as the establishment of ocular dominance columns. Studies on subplate neurons in primate cortex development should also include frontal and parietal association areas where IN are known to be very numerous in the adult.

\section{A commentary on}

The changing roles of neurons in the cortical subplate by Friedlander, M. J., and Torres-Reveron, J. (2009). Front. Neuroanat. 3:15. doi: 10.3389/neuro.05.015.2009.

\section{REMARKS AND MAIN CONCLUSIONS}

1. We propose that certain types of neurons can undergo a temporal re-specification of function over the lifespan. Specifically, we suggest that the population of cortical subplate neurons does so although it is not known whether individual neurons in that cohort of cells change their function or if the surviving subplate cells represent a subpopulation that has different functions at different stages of the life cycle.

2. Although we propose a long term type of multi-tasking over the lifespan, there may be other types of neuronal multitasking operating over shorter time scales. For example, within minutes, as information is being processed, neuromodulators and recent bouts of activity could unmask emergent functional properties such as regulation of gene expression leading to differential functions of individual neurons within the neuronal circuit within which the cell is embedded. Recording of electrical activity from large populations of interacting neurons will be required, while following individual neurons' activity profiles for extended periods, with tetrode arrays to directly test these ideas. Current technology can apply in vivo optical monitoring of the dynamics of the structure of dendrites, spines and axons 
although this approach is generally limited to superficial cortical layers. New advances in imaging technology will be required for similar tracking of deep cells such as subplate neurons during development. It will also be of interest to evaluate whether changes in neuronal function occur over the course of aging. For example, the neocortex shrinks during normal aging due primarily to atrophy of cells and the neuropil. Future studies of individual cellular and neuronal network function during aging may reveal other examples of serial neuronal multi-tasking.

3. Differential expression of genes plays a major role in neuronal development and functional differentiation not only from early embryonic stages but also into senescence. These changes can be programmed to occur at defined stages or can be triggered by local signals, by environmental inputs or in a neuronal activity-regulated manner. Such temporally modulated regulation of gene expression can play a role in target recognition and path-finding, synaptogenesis, refinement of synaptic connections. In addition, other influences such as sensory or motor activity, cognition, stress, infectious agents and traumatic events can alter gene expression patterns in the brain throughout life.

4. After differentiation to a particular phenotype, little change is thought to occur in each neuron's fundamental properties such as their anatomical projections, location, position, chemical neurotransmitter, and the functions of the cell within the framework of the particular network where it resides.

5. Pleiotropy (the ability of a single gene to influence multiple phenotypic traits) is well established. Neurons can express pleiotropic genes or respond to pleiotropic gene products at different times throughout an organism's life, potentially increasing information processing ability longitudinally and responding to stimuli and stressors. Like genes, whole neurons could increase their information processing contribution combinatorially by serving different functions over the course of the lifespan - a pleiotropy of cellular function in the temporal domain. The neurons of the cortical subplate are one candidate population of cells that may behave in this manner.

6. The subplate cells emerge from the ventricular zone under the cerebral cortex, migrating below the marginal zone to the cortical preplate that is then split by the differentiating neurons of the cortical plate, some neurons taking up residence in the marginal zone and others settling below the cortical plate in the subplate (SP). The cortical plate neurons form most of the cortical layers (layers 2-6) while the marginal zone neurons become layer 1 and the SP neurons become interstitial cells of the cortical white matter as well as clustering at the bottom of the cortical plate just below layer 6.

7. SP cells are among the first cortical neurons to differentiate into a neuronal phenotype; they express microtubule associated protein-2 and neuropeptides before the cortical plate neurons, they receive synaptic inputs and generate action potentials through embryonic development. These cells also serve as pioneers issuing axons into the internal capsule where they serve an important role by innervating the thalamus and providing a scaffold for the innervation of the cortex by the thalamocortical axons.

8. The SP neurons are also transiently innervated by the ingrowing thalamocortical axons before the eventual thalamocortical target neurons within cortical layer 4 settle in their ultimate positions in the cortical plate to receive their innervation. Layer 4 neurons receive innervation by both SP neurons and thalamic axons during this period followed by removal of inputs from the SP cells through a competitive process. If the SP cells are lesioned, the thalamic axons fail to innervate their correct target areas and cortical columnar organization does not develop normally. Thus, these neurons contribute to establishing functional cortical architecture during development. After performing those functions, most of these cells die.

9. The SP neurons appear to play an important but fleeting role in orchestrating early cortical development. However, although most of these cells die soon after the innervation of the cortical plate by thalamic axons and the retraction of the SP neurons' axons that innervate layer 4, many of them (10-20\%) survive. These cells remain throughout development into adulthood as a compressed band along the bottom of layer 6 (cortical layer $6 \mathrm{~b}$ or cortical layer 7 or subgriseal cells) and as dispersed interstitial neurons scattered in the white matter. It is a matter of considerable interest to know the fate of this group of surviving cells - are they quiescent, do they serve a role in guidance in the postnatal brain as they did prenatally or do they take on an entirely new function? If they change their function and/or connectivity, this suggests a form of temporal pleiotropy for these cells. As these SP cells are greatly reduced in number during development, it is possible that they serve no major functional role after this period. However, this seems unlikely and there are other examples of numerically small neuronal types that contribute in important ways through processes such as numerical expansion of target innervation by axonal and synaptic divergence; strategically positioned or particularly strong synaptic outputs (and/or potent neuromodulatory outputs). Thus, the fact that many of these cells are lost during development should not exclude the possibility that the remaining population of these cells, although relatively small in sheer number, may play some additional important role in cortical information processing. In order to evaluate such a hypothesis, it is necessary to evaluate directly the anatomical and electrophysiological properties of this reduced cohort after their initial role in cortical development and after the elimination of the majority of the cells have occurred.

10. SP cells have been shown to be particularly susceptible to or play a role in the pathogenesis of disorders including early neonatal hypoxic-ischemic injury, trisomies, microcephaly and seizures. Although their potential role in such diseases has been studied, there are few studies of the functional properties of the surviving subplate neuronal population in the normal brain, likely due to their sparseness and location, which make such studies difficult. These surviving cells express 
markers typical of neurons including MAP-2 and NeuN and many express the synthetic enzyme for the production of nitric oxide (NO), nitric oxide synthase that can be visualized as NADPH diaphorase (NADPHd) activity. Not only the somata and dendrites are positive for NADPHd but that there is also considerable staining of fine processes and varicosities, suggesting the possibility that these cells may provide a diffusible signal (NO) in the white matter that could play a role in plasticity and/or pathogenesis.

11. Retrogradely transported tracers applied to the surface of the cortex (layer 1) backfill surviving WM and SP neurons' somata, indicating that their axons reach the cortical surface. This projection as well as the presence of boutons on their axons in other cortical layers have been demonstrated for individual surviving white matter and subplate neurons, where their axonal arborizations are visualized by intracellular single cell filling. These neurons also issue axon collaterals within the white matter and deep layer 6, providing the neuroanatomical substrate for them to play a role in a local functional neuronal network.

12. Surviving SP neurons generate action potentials; they receive both excitatory and inhibitory synaptic inputs; and they respond to sustained membrane depolarization with minimal spike frequency adaptation. Thus, these cells retain a neuronal phenotype, they receive synaptic inputs from other neurons and they innervate the various cortical layers. We have also recently found that these cells provide glutamatergic excitatory synaptic inputs to neurons in cortical layer 6 .

13. Interestingly, GABAergic white matter neurons with projection axons have been identified in primates and we have seen a subset of GABAergic WM and SP neurons in rat using immunohistochemistry, although we have yet to record from an identified surviving presynaptic GABAergic neuron. In addition to having fast glutamatergic excitatory synaptic output, these cells also stain positively for various neuromodulators including substance $\mathrm{P}, \mathrm{CCK}$, somatostatin and nitric oxide synthase (NOS). The diversity of secreted chemicals that these surviving cells contain together with their capacity to maintain protracted non-decrementing trains of action potentials in response to a sustained depolarizing drive may afford these surviving neurons the capacity to provide strong neuromodulatory effects to cells in the overlying cortex.

14. That these cells remain as neurons but also have the capacity to play different roles at different stages of development is suggested by several factors. These include the persistence of intrinsic electrophysiological and synaptic properties, survival of the glutamatergic phenotype, receipt of excitatory and inhibitory synaptic inputs from other sources after the loss of their thalamocortical inputs and the re-arrangement of their axonal outputs from transiently innervating layer 4 to innervating all cortical layers. Much of the information about the properties of these surviving cells must, by necessity be obtained from in vitro brain slice preparations so there is little known about their properties within the circuitry of the intact brain. While their basic electrophysiological properties can be studied in the brain slice preparation, features such as how they process sensory information or identifying the sources of their synaptic inputs from distant sites are difficult to determine in vivo, since the cells are sparse (WM interstitial cells) or compressed in a thin sheet (the subplate cells at the bottom of layer 6 or subgriseal cells). Thus, although we now know somewhat more about the intrinsic and local synaptic properties of these cells in the postnatal brain, their precise function within the mature cortical network must remain somewhat speculative.

15. The surviving group of SP neurons may function as a sort of cortical gatekeeper, modulating information flow into and out of modules of overlying cortex to other cortical sites. Neurons of the nucleus reticularis thalami (NRT) proximal to thalamic nuclei carry out a similar function as a scattered cohort of GABAergic neurons that are embedded within the internal capsule and receive collateral excitatory innervation from thalamocortical axons as well as from corticothalamic axons and provide connectivity to each other within the NRT. They innervate thalamic neurons in inhibitory feedback projection from the thalamus and provide an inhibitory feed-forward projection from layer 6 of the cortex, as well. The NRT cells can modify the information processing state and the relay of information from the sensory periphery to the cortex by modulating membrane potential. The cohort of surviving white matter and subplate neurons may perform a related function in the cortex.

16. The dendritic arborizations of the WM and SP neurons within cortical layer 6 position them strategically to receive synaptic input that is otherwise destined for layer 4 from collaterals of thalamocortical axons that also arborize in layer 6. In addition, they could also receive synaptic input from the axons of cells in cortical layer 2 and 3 that send axon collaterals to layer 6 and even into the white matter. That is, these neurons could also receive a copy of information that has been processed within the cortical columnar structure and is being relayed to other cortical areas.

17. Although their somata are located within the white matter, many of the interstitial white matter neurons also have dendrites located in layer 6 where they are also positioned to potentially receive similar synaptic inputs. A difference between these neurons and the NRT cells [see point 15] is that most of the surviving subplate and many white matter neurons are glutamatergic vs. GABAergic. However, it is interesting to note that a substantial fraction of the subplate neurons are GABAergic, although we apparently have only recorded from the glutamatergic ones in our paired recordings since in all cases, the postsynaptic response was excitatory. It is not clear why our recordings should select only the glutamatergic neurons in the SP but because our results are so far limited to that sub-population, the multi-tasking behavior of these cells might be limited to certain subsets.

18. The excitatory synaptic output of the SP and WM neurons to the cortical layers above could provide either feed-forward (for the thalamocortical inputs) and/or feedback (for the cortical efferents) information. Since the surviving SP neurons are mostly excitatory and they innervate neighboring SP cells in addition to the overlying cortical neurons, they 
could act as an amplification network for important signals through activating of a local network of neighboring liketype cells as well as a subset of postsynaptic targets in the overlying cortex. Recurrent excitation in such an arrangement could however, create network instability or seizures but depending on the types of cells that are targeted (e.g. glutamatergic excitatory vs. GABAergic inhibitory neurons), the properties of such a circuit may allow for selective amplification and contrast enhancement through feedback inhibition. The neuromodulatory chemicals in the SP and WM neurons such as NOS and various neuropeptides such as substance $P$ further enhance the potential of these cells' output functions through signal gating or selective amplification that could be useful for attention, sensory learning by enhancing signal to noise ratios or changing activation thresholds and synaptic integration properties of neurons within the cortical network.

19. Summary. There still remain considerable issues to be resolved regarding the role of these intriguing SP neurons within the mature neocortex. For example, how do the surviving cells avoid elimination during development? Which cells provide the synaptic inputs to these neurons? What are the functional properties of these neurons in vivo? What role do the many neuromodulators released by these cells play in information processing? Do these surviving white matter and subplate neurons retain the capacity to re-enable early developmental processes in the adult cortex after injury or disease? The answers to many of these questions must await experiments where these cells are studied in the adult brain in vivo with selective targeting techniques. However, it is clear that subplate neurons perform important functions in the cortex during early development and that a substantial number of these cells organizes into a different functional network in the postnatal brain that could contribute to cortical function in other ways. Whether such longitudinal pleiotropy of neuronal phenotype applies throughout the lifespan to the aging brain and/or to other neuronal populations remains to be evaluated. If so, this would dramatically enhance the capacity of neuronal networks throughout the lifespan.

\section{GENERAL COMMENTS AND DISCUSSION ROCKLAND}

As the authors point out (point 9 and elsewhere), the terminology for subgriseal and white matter neurons is not standardized. Even in rodent, the same neuron population can be called "layer $6 \mathrm{~B}$," "layer 7," or subgriseal. This important issue is likely to be more extensively addressed in the following Special Topic, on GABAergic cortical projection neurons.

\section{ROCKLAND}

The possibility of pleiotropy of cellular function in the temporal domain - serial lifespan related and/or short-term (activity related?) neuronal multi-plexing - is provocative (points 2, 5, and elsewhere). In further investigations of this point, the sparseness of this population may actually be advantageous, if cell-type specific markers can be functionally exploited.

\section{ROCKLAND}

The authors very legitimately note that strength is not always in numbers (point 15), and that this relatively sparse population may still be exerting a significant influence.

\section{KOSTOVIC}

It is interesting that subplate neurons form a well delineated cytoarchitectonical layer in rodent brain-a compressed band along the bottom of layer 6 (remark 6). This cyto- architectonical correlate of the subplate feature may be explained by major differences in organization of white matter between rodents and primates: simplified corona radiata and absence of gyri in rodents. However, this also indicates a more uniform population of subplate neurons and a restricted developmental origin in rodents. The article makes very good points about the changing role and different function of subplate neurons over the course of the lifespan (remark 5), and the involvement of the subplate in the generation of action potentials. These factors will elaborate the explanation of the developmental roles, as will be presented in the next Specific topic "Cortical GABAergic Projection Neurons"

\section{A commentary on}

Individual differences in distinct components of attention are linked to anatomical variations in distinct white matter tracts by Niogi, S., Mukherjee, P., Ghajar, J., and McCandliss, B. D. (2010). Front. Neuroanat. 4:2. doi: 10.3389/neuro.05.002.2010.

\section{REMARKS AND MAIN CONCLUSIONS}

1. White matter tracts provide the anatomical connectivity essential for normal cognitive functioning that requires the integration of neural computation across spatially separated cortical regions such as attention and executive function abilities. This has potentially strong implications for understanding how variations in structural properties of white matter tracts from one person to another may systematically influence individual variations in efficiency across a wide range of cognitive domains, even within healthy individuals exhibiting no signs of neural or cognitive dysfunction. In support of this notion of a dimensional structure-function relationship between white matter tract microstructure and cognitive abilities, an increasing number of diffusion tensor imaging (DTI) studies have shown that individual differences in white matter microstructure are systematically linked to individual differences in cognitive domains including reading and phonological processing, numeracy and mathematical abilities, executive attention, visual attention, alerting, and memory. Additionally, it has been demonstrated that reaction time measures are sensitive to detecting variations in efficiency of cognitive domains.

2. Moreover, a growing body of evidence suggests that DTI assessments taken from different white matter tracts may correlate specifically with performance in different cognitive domains. As an example, DTI measures from two distinct white matter tracts in the same population of subjects have been found to correlate with performance in two distinct cognitive domains. We showed that within the same population correlations exist between reading ability and frac- 
tional anisotropy (FA) in a left superior-inferior fiber tract, but these measures were unrelated to a similar structurefunction correlation between short term memory and FA in a frontal association tract. Similarly, double dissociation findings within a single population of normal healthy adults demonstrating structural and functional specificity were found for the relationships between attention and FA in the anterior corona radiata (ACR) versus long term memory formation and the uncinate fasiculus (UF). Such findings serve to establish that specific, separable structure-function associations can be found across different neural networks, and variations in white matter tract properties are often closely linked to variations within these distinct cognitive domains. Such individual differences studies have, however, yet to examine whether such structure-function distinctions might also hold true for components within a specific cognitive domain.

3. Attention is a complex cognitive domain that has been extensively investigated by employing cognitive paradigms attempting to isolate functional components as well as by employing neural studies to investigate their associated brain systems. Although many competing theories have proposed a number of potential components of attention, one highly influential approach has proposed that three broad functional distinctions can be made that account for a multitude of findings across cognitive studies, neuropsychological investigations, and neuroimaging studies. Posner and Petersen proposed that investigating attention in terms of three separable component functional processes - alerting, orienting, and executive function - would help integrate a host of cognitive and neural investigations. Over the last two decades, these components of attention have been linked to separable brain networks via functional neuroimaging, electrophysiology, and lesion studies.

4. The efficiency of the individual components of the attention system proposed by Posner and Petersen can be separately measured using the Attention Network Test (ANT). The ANT is a reaction time task that measures the latency to decide whether a specific arrow symbol points leftward or rightward (the imperative stimulus). The ANT is designed to examine how such response times are impacted by different components of attention. To manipulate the alerting component of attention, the imperative stimulus which otherwise appears after a random time interval is preceded by a visual warning cue to alert the subject that a stimulus is about to appear. To manipulate the orienting aspects of attention, the location of the imperative stimulus which is otherwise unpredictably either above or below the central fixation cross is revealed in advance by a spatial cue that orients the subject's spatial attention to the correct location. The executive component of attention is manipulated by introducing or removing conflicting irrelevant information; the imperative stimulus is presented along with "flanking" arrows on either side that either point in the same direction or in the incongruent (conflicting) direction. Examining how these three classes of manipulations (alerting, orienting, and conflict) impact response times provides an assay for the efficiency of each of these three components of attention. Given the simplicity and sensitivity of the
ANT to three largely independent components of attention, it has been used in children and adults in normal populations, and in cohorts with neuropsychiatric disorders such as ADHD, schizophrenia, and borderline personality disorder.

5. By approaching the human attention system as one comprised of several separable networks, one can create paradigms in which they operate in a fairly independent fashion in order to examine them individually. Alternatively, different paradigms may prove useful in studying the ways in which these systems interact.

6. Functional and neuropsychological studies have associated performance in conflict tasks (executive control) to the frontal cortex, and more specifically to a network including the anterior cingulate gyrus and lateral prefrontal cortex. The conflict component of attention mediates inhibitory control, resolution of conflicting stimuli impacting decision making, and, in a broader sense, can be considered necessary for decision planning and decision making. This network likely includes white matter tracts that serve to connect these regions with other structures.

7. The frontal lobes contain numerous complex connections with different parts of the brain, many of which pass through the thalamus. As such, it is likely that tracts from the thalamus extending to the frontal lobe and anterior cingulated gyrus, such as the ACR, may be part of the executive attention network associated with the conflict component of the ANT. Indeed, a recent study demonstrated that white matter integrity along the left ACR correlated significantly with conflict performance from the ANT in a group of normal adults and also in a cohort of adults with mild traumatic brain injury.

8. The alerting component of attention is proposed to be responsible for activating the required cognitive systems to make the person ready to respond to a task. This form of phasic alerting is modulated by thalamic, frontal, and parietal regions. Although the reticular activating system is known to be necessary for tonic alertness, it also plays a critical role in phasic alerting. A likely white matter pathway that connects the proposed regions critically involves the internal capsule. The internal capsule is made up of an anterior limb (ALIC), and posterior limb (PLIC) and the bend between the two limbs referred to as the genu. The ALIC and PLIC directly relay motor and sensory information with ascending and descending fibers between the cerebral cortex and the pyramids of the medulla.

9. The orienting network selects spatial and sensory information. Commonly, this is tested with visual cues indicating the location of an impending target (as in the ANT). The visual orienting system has been associated with brain areas such as the superior and inferior parietal lobes, frontal eye fields, and subcortical areas including the superior colliculus and reticular nuclei in the thalamus. In considering white matter tracts likely to modulate the efficiency of the orienting network, the optic radiations relay visual information via neurons from the lateral geniculate nucleus of the thalamus to the visual cortex. Such geniculocortical circuitry has been implicated in attention studies. Additionally, the orienting system must relay and compare spatial infor- 
mation from both visual fields which requires connectivity between hemispheres. Lesions studies of the splenium of the corpus callosum, fMRI studies of interhemipsheric transfer, and studies examining callosal thickness in ADHD suggest that commissural fibers, particularly the splenium of the corpus callosum may play a large modulatory role in the function of the visual spatial orienting network.

10. Prior neuroimaging studies focused primarily on functional activations using fMRI or EEG to isolate the anatomic substrates for the attention networks. These studies have focused on the functional activations (i.e. gray matter) involved in the attention components. It remains unclear what specific white matter pathways modulate each component of attention. As has been demonstrated in several other domains reviewed above, individual differences in white matter microstructure within tracts associated with particular attention networks may closely correlate with variations in efficiency of these attentional processes.

11. One technique to quantify white matter integrity is DTI. The principle governing DTI is that water diffuses more readily along the orientation of axonal fibers than across the fibers due to hindrance from structural elements such as the axolemma and the myelin sheath. The degree of directionality is termed anisotropy. Anisotropy can be measured as the variation in the eigenvalues of the diffusion tensor. Fractional anisotropy (FA), a normalized measure of anisotropy, has been shown to be sensitive to microstructural changes in white matter integrity and organization. Increasing numbers of DTI studies that correlate FA with cognitive function indicate that such measurements can be used to account for a wide range of cognitive skill.

12. The Conflict Network. The correlation analysis together with the multiple regression dissociation analysis provides evidence that microstructural integrity of the ACR modulates executive attention. The finding of a frontal tract associated with executive attention is strongly supported by previous literature. Functional imaging studies have provided strong consistent support for the notion that the frontal lobes, particularly the anterior cingulate gyrus, is associated with attentional control during the Stroop Task and the conflict portion of the Attention Network Task. Additionally, neuropsychological data clearly highlight the central role of the frontal lobes in executive attention. Previous DTI studies have also implicated frontal white matter tracts with attention performance. For example, integrity of white matter tracts associated with brain activity impact executive attention. Furthermore, we demonstrated that a closely related frontal executive skill, short term memory, also demonstrated strong correlations with ACR in children. Finally, in a recent study of 43 patients suffering from mild traumatic brain injury, the left ACR integrity assessed by FA was shown to correlate with conflict scores on the ANT. It is interesting to note that in the current study, significant correlation between conflict scores and FA did not reach significance in the right ACR, but appeared only as a nonsignificant trend. Given this trend, and no significant finding of a direct effect of laterality it is possible that both left and right ACR regions play a role in conflict, and the specific contributions of right ACR may be apparent in studies with greater power.

13. The Orienting Network. Regarding the orienting network, the correlation analysis together with the multiple regression dissociation analysis demonstrates that microstructural integrity of the splenium of the corpus callosum modulates the efficiency of the orienting component of attention. This finding is consistent with previous findings that demonstrate that the visual-spatial attentional orienting system is dependent on a functional network that includes left and right posterior parietal regions, and is linked to a larger neural network including frontal eye fields, and subcortical areas including the superior colliculus and reticular nuclei in the thalamus. We propose that the splenium of the corpus callosum includes white matter tracts involved in this overall network, and that this region provides a large convenient homogenous white matter region of interest that can be used to assess individual differences in this network. Our more specific findings implicating the splenium of the corpus callosum as part of the network that modulates orienting performance is consistent with Noudoost and coworkers' report of a case study that demonstrates the role of interhemispheric connections in making an integral visual map across hemifields that can be used for visual spatial attention. Furthermore, splenium of the corpus callosum lesions commonly cause visual spatial neglect, a deficit that specifically impacts orienting functions of attention. Such lesions can also cause other forms of disconnection syndromes that may be linked to spatial orienting functions as in the case or hemialexia.

14. The Alerting Network. The correlation analysis together with the multiple regression dissociation analysis provides evidence that the PLIC modulates individual differences in the alerting component of attention. This relationship is generally consistent with the previous literature suggesting a role of the PLIC for this component of attention. For example, Sturm and coworkers presented PET evidence from normal volunteers that suggested alerting functions involve a vast network of regions, including inferior parietal-thalamic networks, brainstem structures, and frontal regions, largely lateralized to the right hemisphere. Fimm and coworkers investigated alerting related impairments of attention in 15 patients with acute circumscribed vascular lesions confined to the basal ganglia, internal capsule, and thalamus. In his study, five out of seven patients showed evidence of lesions to the PLIC of the internal capsule. Fimm and coworkers suggested that thalamo-parietal projections transversing through the PLIC via the superior peduncle of the thalamus could lead to a disconnection of functionally relevant structures that impair attention.

15. Next, we turn consideration to the separability hypothesis regarding the three attentional networks. In evaluating these specificity findings, it is important to consider that the ANT was specifically designed to stress the potential conditions under which the three attention networks could be shown to be separable in their operations and individual difference 
patterns. More recently, several investigations have begun to explore other conditions under which these networks might interact and influence one another in important ways. To the degree to which the ANT exposes these networks to be anatomically and functionally separable, it is possible that white matter tract integrity is an important independent modulator of function, such that individual differences in white matter tract microstructure in one network might be functionally associated with one component of attention relative to the other components of attention. This hypothesis was addressed by a series of multiple regressions, in which the variance in function for each established white matter tract ROI was assessed for the key proposed associated function, while controlling for the influence of the other component attentional functions.

16. Results of this multiple regression analysis demonstrated three key findings: the ACR is a unique modulator (relative to the other two ROIs) of the conflict network function, the PLIC is a unique modulator of the alerting network, and the splenium is a unique modulator of the orienting network. This correlational triple dissociation can be interpreted similarly to the "gold-standard" double-dissociation tests in neuropsychology commonly required when attempting to establish specificity of structure-function relationships. Such findings are critical in lesion studies in which the mere presence of any form of brain damage can cause widespread, non-specific difficulties in any performance assessment of cognitive function, and establishing specificity of functional and structural loss is critical. Similarly, in linking individual differences between white matter properties and cognitive performance, establishing similarly high levels of specificity between particular regions and particular functions is critical.

17. In addition to showing functional specificity, the correlational dissociation analysis shows that within each functional network, a gradient of individual differences in function positively correlates with a gradient of individual differences in white matter tract microstructure. Furthermore, since this study focused on normal healthy adults, rather than reflecting the impact of neural damage on related loss of function, the results may be more relevant to understanding normal population variations in white matter tract microstructure and how such differences are linked to efficiency of components of attention. As such, these findings may provide the basis of relating dimensional differences in structure-function relationships that exist within normal populations with more extreme ranges of variation of structural damage and related loss of function.

18. In conclusion, combining these observations with previous fMRI and neuropsychological studies suggests the components of attention are comprise of segregated functional networks and that individual differences in white matter tract microstructural integrity might modulate these functionally specific neural networks. Thus, understanding individual differences in white matter tract microstructural integrity may prove an important complement to fMRI studies of functional organization of cortical function.

\section{GENERAL COMMENTS AND DISCUSSION HÖISTAD AND HOF}

In regard to point 12, a recent fMRI study (Fan et al., 2008) using the ANT and examining physiological response of several brain regions in terms of interactions between conflict processing and activity of the anterior rostral cingulate cortex, and the effective connectivity between it and other cortical domains using psychophysiological interaction analysis and dynamic causal modeling showed a significant integration of the anterior cingulate with the caudal cingulate zone of the ACC and the lateral prefrontal, primary, and supplementary motor areas above and beyond the main effect of conflict and baseline connectivity. The intrinsic connectivity from the anterior to the caudal cingulate cortex was modulated by the context of conflict, indicating that conflict processing is associated with the effective contribution of the rostral cingulate to the neuronal activity of the caudal cingulate cortex, as well as other cortical regions.

\section{A commentary on}

The effects of normal aging on myelinated nerve fibers in monkey central nervous system by Peters, A. (2009). Front. Neuroanat. 3:11. doi: 10.3389/neuro.05.011.2009.

\section{REMARKS AND MAIN CONCLUSIONS}

1. There are two types of nerve fibers in the central nervous system, myelinated and unmyelinated ones. The myelinated nerve fibers are axons of neurons that are ensheathed by internodal lengths of myelin formed by oligodendrocytes. Developmentally, the internodal lengths of myelin are produced at the ends of processes of oligodendrocytes and each internode is generated by a spiral wrapping of a paired sheet of oligodendrocytic plasma membrane. Initially the successive turns of the spiral of paired membrane sheets are separated by cytoplasm, but eventually the cytoplasm is extruded from between the turns. As a result, mature, compact myelin is formed. At the ends of each internodal length of myelin are regions called paranodes, and here the turns of the spiral wraps of myelin membrane successively terminate, the innermost one terminating first. As the turns of myelin terminate, the sheath gradually becomes thinner, and eventually ends at the nodes of Ranvier, which separate the successive internodal lengths of myelin. At the nodes the axon is bare, but possesses a dense undercoating.

2. An oligodendrocyte forms several internodal lengths of myelin, each one on a different axon, and in general the larger the diameter of the axon, the thicker is its myelin sheath and the longer its internodes and its paranodes. And since there seems to be some limit to the amount of myelin an individual oligodendrocyte can produce and maintain, oligodendrocytes that myelinate small diameter axons form more internodal lengths of myelin than those that myelinate larger diameter axons.

3. Myelin contains lipoproteins, so that in unfixed brains the myelin sheaths have a white sheen. Consequently, tracts of the central nervous system that contain mostly myelinated nerve fibers and few neurons are referred to as white matter. In contrast, gray matter contains the cell bodies and dendrites of neurons and fewer myelinated nerve fibers. 
4. The first hint that there are age-related changes in myelinated nerve fibers came from the observation that in old humans and monkeys there is a decrease in the intensity of haemotoxylin staining of white matter. The underlying reason for this increased staining pallor is still not clear, but it is now know that there are a number of age-related alterations of myelinated nerve fibers in the primate central nervous system, such as a loss of some myelinated nerve fibers and alterations in the morphology and composition of myelin sheaths, that could account for the decrease in staining intensity.

5. Magnetic resonance imaging (MRI) studies of both human and monkey brains have shown there is a loss of white matter from the cerebral hemispheres with age.

6. Over the life span of the monkey the average number of myelinated nerve fibers lost from the optic nerve and from the anterior commissure is about $45 \%$, while from the fornix and the splenium of the corpus callosum, the loss is about $25 \%$. In all four structures the correlations between the decreasing numbers of myelinated nerve fibers and increasing age are significant.

7. In contrast, there is no measurable loss of myelinated nerve fibers from the visual cortex, but the inability to detect a loss may be due to the relatively sparse numbers of myelinated nerve fibers present in cortex, because a few myelinated nerve fibers with degenerating axons have been seen in cortex. Indeed myelinated nerve fibers with degenerating axons, as indicated in the electron microscope by the presence of dense axoplasm with a loss identifiable organelles, or the presence of empty myelin sheaths, have been encountered in all of the parts of the aging monkey brain that we have examined, suggesting that myelinated nerve fiber loss is ubiquitous. And based on earlier studies of Wallerian nerve fiber degeneration, there is little doubt that once an axon degenerates, breakdown and degeneration of its myelin sheath inexorably follows.

8. Myelinated nerve fiber loss from white matter in pathways must result in some disconnection between various parts of the central nervous system. But interestingly, although there are no significant correlations between the extent of myelinated nerve fiber loss from the splenium of the corpus callosum and the cognitive decline shown by monkeys, there are correlations between cognitive decline and myelinated nerve fiber loss from the anterior commissure and the fornix. In this context, it is interesting that cutting the splenium of the corpus callosum, which is the principal fiber pathway connecting the occipital cortices, has little effect on cognition. The anterior commissure provides the interhemispheric connection between the entire temporal lobe, as well as parts of the orbitofrontal cortex, prepiriform cortex and the amygdalas, and numerous studies have shown that the anterior commissure provides a pathway whereby visual information can reach the opposite hemisphere and contribute to behavioral responses, such as two-choice discrimination. The fornix, on the other hand, carries the main output from the hippocampus, and studies of the effects of lesioning the fornix in both monkeys and humans have revealed the role of the fornix in memory and have described amnesia as a major consequence of making such lesions.
9. It might be assumed that since myelinated nerve fibers are lost from white matter with age, that there must be a concomitant loss of the neurons from which the nerve fibers arise. For the optic nerve, this may be the case, since retinal ganglion cells are subject to damage from ocular changes and systemic disease that occurs frequently in the elderly. But for the other central nervous system pathways, in which the myelinated nerve fibers arise from cortical neurons, a different reason has to be sought, because, as stated above, recent studies have shown that in normal aging few neurons are lost from the cerebral cortices of either monkeys or humans. Freeman and coworkers have recently shown that in normally aging humans, cortical neuron numbers are preserved even when there is cortical atrophy. To account for the age-related loss of myelinated nerve fibers from white matter, we have suggested that only the portion of the axonal plexus of a pyramidal cell that enters the white matter, degenerates by a dying back process, leaving the more extensive local axonal plexus in the cortex intact. This scenario would account both for the loss of some myelinated nerve fibers from white matter and for the failure to detect myelinated nerve fiber loss from the cerebral cortex itself.

10. Obviously, in normal aging some myelin sheaths degenerate as a consequence of their axons degenerating, but in other cases myelin sheaths degenerate even though the axon is intact. In the latter category there are two kinds of myelin sheath alterations. The most common age-related degenerative alteration is an accumulation of dark cytoplasm in pockets that are produced by a splitting of the major dense line The location of the dense cytoplasm in splits of the major dense line implies that the cytoplasm must be derived from the parent oligodendrocyte, because the major dense line of the myelin sheath is produced by apposition of the cytoplasmic faces of the plasma membrane of the oligodendrocyte forming the myelin sheath.

11. Proof of the fact that the accumulation of dense cytoplasm in normal aging is a degenerative change comes from studies of cuprizone toxicity, which leads to oligodendrocyte death. This results in the formation of dense cytoplasm in the cytoplasmic process on the inner face of the myelin sheath.

12. Another, but less common myelin alteration associated with aging is the formation of myelin balloons. These balloons can be as large as $10 \mu \mathrm{m}$ in diameter, so that even by light microscopy the larger balloons are visible as holes in the neuropil of the aging cortex. Electron microscopic analyses show that these holes are really localized fluid-filled cavities that are accommodated by splits in the intraperiod line of the affected sheaths, and since the intraperiod line is produced by apposition of the outer faces of the cytoplasmic membrane of the oligodendrocyte, the fluid-filled sacs are potentially in contact with the extracellular space.

13. There is evidence that the formation of balloons is a degenerative process, since myelin balloons can be produced by cuprizone and tetraethyl tin toxicity, and by chronic copper poisoning. Balloons can also occur in early phases of Wallerian degeneration and in severe diabetes. 
14. When the percentage of myelinated nerve fibers showing either the presence of dense cytoplasm or of balloons is examined, it is found that the frequency of such profiles increases significantly with age. More importantly, there are significant correlations between cognitive declines and the frequency of profiles of degenerating sheaths in cortical area 46 , splenium of the corpus callosum, anterior commissure, and fornix. An exception is primary visual cortex, in which there is no correlation between cognitive decline and myelin sheath degeneration. This may be because primary visual cortex has little role in cognition. It is presumed that the correlations between myelin degeneration and cognition are due to the degeneration resulting in a slow down in conduction velocity. This would adversely affect the timing in neuronal circuits.

15. Duce and coworkers have identified a number of genes that might produce cytotoxicity in white matter. These genes range from ones that can affect life span, to ones that can affect the reorganization of glial cytoskeleton. Others can produce oxidative and proteolytic injury, and yet others are cell cycle inhibitors. But these authors focus particular attention on a gene called Klotho, a multifunctional gene that is known to defend against oxidative stress, and suggest that with a decrease in the activity of Klotho there is a loss of this protection, which may result in the death of oligodendrocytes.

16. There are other age-related alterations in myelin sheaths, which indicate that myelin continues to form with age. The first is an increase in the overall thickness of normal myelin sheaths with age. However, the increase in thickness of sheaths is not uniform. The mean increase in the numbers of lamellae is largely because thick sheaths, with more than 10 lamellae, become more common in old monkeys.

17. Another change that is considered to indicate the continued formation of myelin is the formation of sheaths that contain redundant myelin, so that the sheaths are too large for their enclosed axons. When such sheaths are cross-sectioned and examined by electron microscopy the axon is seen to be located at one end of an excessively large sheath that loops off into the surrounding neuropil.

18. When the frequencies of various kinds of profiles of myelinated nerve fibers are quantified in the vertical bundles of nerve fibers in the cerebral cortex, it becomes evident that the frequency of profiles of paranodes increases with age.

19. We have not been able to identify demyelinated nerve fibers in the monkey brain, but this should not be a surprise, since such demyelinated nerve fibers would be expected to resemble unmyelinated nerve fibers. However, in support of the fact that demyelination is taking place, we have seen fragments of degenerating myelin within the cytoplasm of both microglia, and more commonly within astrocytes in the brains of aging monkeys. Also some of the amorphous phagocytosed material within the cytoplasm of astrocytes in the cerebral cortex of old monkeys labels for antibodies to myelin basic protein.

20. In monkey cerebral cortex stained with Perl's reaction for ferric iron the processes of some oligodendrocytes in old monkeys show swellings along their lengths, and when these swellings are examined in the electron microscope it is seen that they contain dense inclusions. Most probably the material is produced by degeneration of some components of the myelin sheaths that belong to the oligodendrocytes, and it is tempting to suggest that the material is related to the dense cytoplasm that accumulates between the lamellae of some sheaths in old monkeys.

21. It is also common in old monkeys to find oligodendrocytes in pairs, rows and groups, suggesting that oligodendrocytes may be proliferating with age, and when comparisons are made between the numbers of oligodendrocytes in young and old primary visual cortices it is evident that there is an increase in the numbers of oligodendrocytes with age. In contrast, there are no changes in the frequency of either astrocytes or microglial cells with age.

22. What is the origin of the increased numbers of oligodendrocytes that are generated, and why are they necessary? The formation of groups and rows of oligodendrocytes during aging could be taken to suggest that oligodendrocytes are dividing, but the prevailing view is that mature oligodendrocytes do not divide, and in a study of the generation of new cells in the adult dentate gyrus of the hippocampus in old monkeys using BrdU labeling no labeled oligodendrocytes were found. It is more likely that new oligodendrocytes originate from the oligodendroglial precursor cells which express NG2 chondroitin sulfate. These cells are scattered throughout the central nervous system, and in adult rodents they account for about $5 \%$ of all neuroglial cells.

23. Moreover, Rivers and coworkers have recently shown that in adult mice many of the newly generated oligodendrocytes that arise from the oligodendrocytic precursor cells during adulthood are involved in myelination. They calculate that about $20 \%$ of all oligodendrocytes in the adult corpus callosum are generated during adulthood and that many of these cells form myelin. In contrast, the same group calculates that only about $5 \%$ of the adult-born oligodendrocytes in the cerebral cortex appear to be involved in the elaboration of myelin sheaths.

24. Unfortunately there is no information about the rate of turnover of oligodendrocytes in the adult monkey, but there is no reason to doubt that it is significantly different from in rodents.

25. A synthesis. It is proposed that the following scenario can explain the available data on the effects of age on myelinated nerve fibers in the central nervous system of the monkey.

a. During aging some neurons lose their long projecting myelinated axons that enter white matter, while retaining their local plexuses so that the parent neuron does not die. The consequence of this is that, as has been demonstrated, some myelinated nerve fibers are lost from white matter, even though there is no significant loss of neurons from the cerebral cortex. For other neurons the effects of aging are less severe, since their axons remain intact, even though some of the internodal lengths of myelin that ensheath them degenerate. 
b. The process of demyelination probably begins as an oligodendrocyte shows stress and starts to accumulate dense inclusions in swellings of its processes and in its perikaryon, as well as in spaces between the lamellae of the myelin sheaths for which the oligodendrocyte is responsible. Ultimately the oligodendrocyte dies, which results in the degeneration and loss of the internodal lengths of myelin belonging to that oligodendrocyte. Oligodendrocyte precursor cells are then activated and generate new oligodendrocytes that repair the damage by remyelinating the bare lengths of axons.

c. In the process of remyelination, several new oligodendrocytes are involved in the replacement of the original internode of myelin. These oligodendrocytes produce shorter internodal lengths than the original one, and the new sheaths are thinner.

d. Thus, when profiles of sectioned myelin sheaths in older monkeys are examined, it is found there is an increase in the number of profiles of paranodes, and this is accompanied by an increase in the total number of oligodendrocytes. This breakdown of myelin sheaths, together with the formation of shorter internodal lengths of myelin and the consequent increase in the number of nodes of Ranvier, would result in a slowing down of the rate of conduction along affected myelinated nerve fibers. Consequently the timing in neuronal circuits would be affected and contribute to cognitive impairment that occurs with increasing age.

\section{GENERAL COMMENTS AND DISCUSSION DeFELIPE}

Comment on point 16:

Is it possible that thin myelinated axons are more vulnerable with age and, therefore, thicker axons may seem to be more common or are there quantitative studies regarding this subject?

\section{ROCKLAND}

I wonder if there is any "intercommunication" or shared signal among the different axons that are myelinated by any given oligodendrocyte? Or, similarly, between those oligodendrocytes that myelinate a given segment of axon (point 2)?

\section{ROCKLAND}

Peters notes several conditions that could be assumed to result in circuitry-significant changes in conduction velocity. In this regard, see Kimura and Itami.

\section{HÖISTAD AND HOF}

There is a loss of white matter (WM) from the cerebral hemispheres with age. Peters presents some of the observed ultrastructural morphological changes that may occur in WM during aging, including myelin balloons, redundant myelin, split sheaths and sheaths with dense cytoplasm. He discusses evidence that redundant myelin may be due to uncontrolled production of myelin with age, while thicker myelin sheaths may be evidence for the continued formation of myelin with aging. Some questions that arise are whether the myelin changes observed in aging are different in WM versus the grey matter, and if age-related changes in the grey matter can be area or layer specific. In addition, how are normal aging alterations in myelin different from those observed in disease, for example in schizophrenia (Uranova et al., 2001). Is there a commonality between normal aging and alterations seen in disease, which could lead to comparable alterations of neuronal communications and result in specific cognitive and behavioral changes?

\section{A commentary on}

Oligodendrocyte development and the onset of myelination in the human fetal brain by Jakovcevski, I., Filipovic, R., Mo, Z., Rakic, S., and Zecevic, N. (2009). Front. Neuroanat. 3:5. doi: 10.3389/neuro.05.005.2009.

\section{REMARKS AND MAIN CONCLUSIONS}

1. The origin and differentiation of oligodendrocytes have been extensively studied in animal models, and are especially well documented in rodents, thanks to advances in various molecular biology techniques that have provided the means of genetic mapping of cell lineages in the developing mouse brain. Along the neural tube oligodendrocytes are produced ventrally under the influence of the morphogen Sonic Hedgehog (Shh), and migrate at the progenitor stage to dorsal regions. It has been shown, however, that after the initial wave of ventrally derived oligodendrocytes, they are completely replaced by a dorsally derived population. In the ventral diencephalon and telencephalon, Shh influences the expression of oligodendrocyte lineage genes, Olig1 and Olig2, which are basic helix-loophelix (bHLH) transcription factors. The question, however, remains to which extent could observations obtained on these animal models relate to human brain development.

2. Early oligodendrocyte progenitor cells are characterized by their expression of platelet derived growth factor receptor alpha (PDGFR $\alpha$ ) and NG2 proteoglycans, and by a typical morphology with few ramified processes. These cells are still mitotic, so we consider them "progenitors". Later along the oligodendrocyte lineage, cells are not considered to be proliferative any more. Thus in this review, we use the term "precursors" for these cell types. We detected the first PDGFR $\alpha$ expressing $\left(\mathrm{PDGFR}^{+}\right)$cells in the forebrain of 10-gw old fetus, but they appear in higher numbers only around $15 \mathrm{gw}$, when they are most numerous in the ganglionic eminences and in the cortical VZ/SVZ. By midgestation (19-22 gw) oligodendrocyte precursor cells invade more dorsal areas of the telencephalic wall, including the cortical plate. During the whole period of our observation (from the onset of neurogenesis, at $5 \mathrm{gw}$, until the beginning of the third trimester, $24 \mathrm{gw}$ ) early oligodendrocyte progenitors were most dense in the cortical SVZ, consistent with their origin from this secondary proliferative zone in the human cortex.

3. In the next stage in oligodendrocyte development, late oligodendrocyte precursor cells are characterized by $\mathrm{O} 4 \mathrm{immu}-$ noreactivity, whereas pre-myelinating oligodendrocytes are reactive to $\mathrm{O} 1$ antibody. $\mathrm{O} 4$ and $\mathrm{O} 1$ antibodies were raised against glycoproteins in the oligodendrocyte membrane and are shown to specifically label cultured late oligodendrocyte precursor cells. Staining of tissue sections with these antibo- 
dies is often hampered by the instability of the glycan epitopes to fixation. Earlier study of rat brain sections has shown that, indeed, it is not fixation but freezing that disperses the antigens and hampers immunostainings. In our studies, however, we have been able to detect $\mathrm{O} 4$ and $\mathrm{O} 1$ expressing cells in human brains when we omitted detergent from the blocking solution.

4. In the human forebrain at midgestation (20-22 gw), we reported that $\mathrm{O}_{4}^{+}$and $\mathrm{O}^{+}$cells are especially dense in the subplate layer, immediately below the cortical plate. This distribution of late oligodendrocyte precursors raises a possibility that transient subplate layer is important for maturation of oligodendrocytes. Considering the importance of a transient subplate layer which receives the thalamic afferents essential for proper wiring of cortical neurons, it is tempting to speculate that oligodendrocytes come to the subplate to obtain yet unknown signals necessary for their maturation and for myelination of the axons in correct sequence.

5. Maturation of oligodendrocytes is marked by the expression of myelin proteins, and the two major myelin proteins, myelin basic protein (MBP) and proteolipid protein (PLP), are the first to be expressed at detectable levels. MBP was detected very early in human embryonic brains (around $5 \mathrm{gw}$ ), but this expression was attributed to Golli/MBP splice variants. In the forebrain, the first $\mathrm{MBP}^{+}$cells of typical oligodendrocyte morphology were found at $18 \mathrm{gw}$, around the middle of intrauterine development. $\mathrm{MBP}^{+}$cells are scattered through the intermediate zone, the future white matter, and increase in numbers with progression of development. Our evidence supports the ventral to dorsal progression of oligodendrogenesis, also reported in rodents. Indeed, a ventro-dorsal gradient in the extent of myelination and in oligodendrocyte precursor cells density was seen in the human fetal forebrain.

6. In the rodent forebrain oligodendrocytes are initially derived from ventrally positioned ganglionic eminences (GE), whereas later they originate from the dorsal cortical SVZ. In human fetal forebrains at midgestation a subpopulation of cortical oligodendrocyte progenitor cells was expressing Dlx2 and Nkx2.1, transcription factors specific for ventrally derived cells in rodents. However, as these transcription factors show a strong signal in the human proliferative zones of both GE and cortex, the origin of human Dlx2- and Nkx2.1-expressing oligodendrocyte progenitor cells could not be simply determined. Another large population of oligodendrocyte progenitor cells at this stage did not express Dlx2 and Nkx2.1 transcription factors, and could represent a population of dorsally derived oligodendrocytes in the human brain. Yet a third population of cells co-labeled with oligodendrocyte progenitor markers (PDGFR $\alpha, \mathrm{NG} 2$, Olig1), stem cell marker nestin, and markers of cells from hematopoietic lineage (CD34, CD68) was present in what appears to be a stream of cells migrating between the GE and the cortical SVZ. These combined findings suggest multiple origins of human cortical oligodendrocytes and have broad implications for normal brain development as well as white matter pathologies.
7. It is possible that oligodendrocytes derived from various sources have different roles, or myelinate different axonal pathways. This becomes especially important and demands further investigation in the light of reports that implicate oligodendrocytes in other functions in addition to "traditional" myelin formation, including synaptic regulation and signaling at the nodes of Ranvier.

8. Studies of the origin of oligodendrocytes in rodents have shown that the ventral morphogen Shh is essential for early specification of oligodendrocyte progenitors in the neural tube. Shh activates a cascade of transcription factors including Pax10, Dlx2, Nkx2, Olig1 and Olig2. Olig2 was described in neural progenitors that give rise to both motor neurons and oligodendrocytes in the ventral spinal cord region. Olig genes belong to a bHLH group of transcription factors that are necessary and sufficient for generation of oligodendrocytes and for myelination in mice. In the human fetal brain we demonstrated the expression of Olig2 in the GE and the medial cerebral cortex starting as early as $5 \mathrm{gw}$, before the onset of either neurogenesis or oligodendrogenesis. Our study also revealed that various cell types later in development express both Olig1 and Olig2. Most notably, Olig2 is expressed in all $\mathrm{MBP}^{+}$cells in the human fetal forebrain and spinal cord at midgestation, and in around $50 \%$ of early oligodendrocyte progenitors in the SVZ. At the same time and at the same location, cortical SVZ, a subpopulation of MAP2 ${ }^{+}$ neuronal progenitors is also Olig $2^{+}$. In contrast, mature neurons in the cortical plate labelled with either NeuN or GABA antibodies, do not co-express Olig2. Taken together these observations suggest the existence of a common progenitor cell for oligodendrocytes and at least some neuronal classes in the human forebrain.

9. Genetic mapping and heterologous transplantation fate mapping studies in mice have shown that the domain within medial ganglionic eminence gives rise to cortical interneurons. There is, however a controversy about the necessity of Olig2 for cortical interneuron fate determination. When Olig2 was ablated from cortical progenitors no change in interneuronal populations was observed. One possible explanation for this finding could be that other bHLH genes, like Olig1, compensate for it. In our study we found Olig1 co-localized in vimentin-expressing cortical radial glia cells, a cell type demonstrated to be a multiple neural progenitor. We have no evidence that in human brain Olig1 is co-expressed in neuronal populations, but that does not preclude the possibility since by the time neurons acquire their fate, this gene may get, down-regulated. This inability to draw conclusions about temporal developmental events is an intrinsic limitation of all studies on fixed human tissue, and is the most important reason why we resorted to different in vitro systems.

10. In vitro, Olig2 plays a role in self-renewal of mouse neurosphere cultures, and in differentiation of neurons and oligodendrocytes in appropriate condition media. In cultures with growth factors (FGF2 and EGF), Olig2 was expressed in almost all progenitors derived from mouse GE or cortex, whereas dorsal transcription factors, Pax6 and Emx1, 
were downregulated. In contrast, in cryosections of human lateral ganglionic eminence at 15-20 gw, Olig2 and Pax6 were co-expressed in the same progenitor cells. This finding was extended to cell cultures from cortical VZ/SVZ. Co-expression of these two transcription factors in dividing progenitor cells is consistent with an increased complexity of human neural progenitor cells in comparison to other mammals.

11. We reported that in the VZ/SVZ of the fetal forebrain, cells occasionally co-express radial glia cells markers and oligodendrocyte lineage markers, suggesting lineage relationship. Radial glia are multipotent progenitor cells in rodents, generating projection neurons and a subpopulation of forebrain oligodendrocytes. Similar to these findings, radial glia cells are also neuronal and oligodendrocyte progenitor cells in human fetal brain.

12. Dorsal transcription factor Pax6 has been reported to be important for generation of neurons in rodents, and also in humans. Pax6 however may also have a role in oligodendrogenesis. Other transcription factors, such as Olig1 and Olig2 may be necessary to specify radial glia as oligodendrocyte progenitors. Indeed we have observed that Olig1 and Olig2 can be co-expressed with the radial glia marker vimentin along the ventricular zone at 15 gw. It would be important to determine whether a majority or only a specific subtype of human oligodendrocytes is generated from radial glia cells and whether radial glia are the only early oligodendrocyte progenitors in the cortical VZ/SVZ. Alternatively radial glia cells could generate oligodendrocytes only at a specific developmental stage or at a particular brain region, such as the cortical VZ/SVZ.

13. Formation of myelin during primary myelination and its restoration in remyelination, involves proliferation, migration and differentiation of oligodendrocyte progenitors into myelin-forming oligodendrocytes. Growth factors, cytokine members of the interleukin (IL) superfamily, chemokines, such as growth-related oncogene alpha (GRO- $\alpha$ ) also referred to as CXCL1, and other mediators influence oligodendrocyte progenitor cells' proliferation and/or differentiation, and are also very important for remyelination. Moreover, it has been demonstrated that this chemokine is upregulated during experimental autoimmune encephalomyelitis (EAE) and around lesions in multiple sclerosis. We studied the distribution of chemokine CXCL1 and its receptor CXCR2 in human developing forebrain, and reported that it is highly expressed in cortical VZ/SVZ in accord with an earlier study in rodents. We demonstrated that in contrast to rodents where CXCL1 directly induces oligodendrocyte proliferation, in human fetal brain CXCL1 has an indirect effect, acting through astrocyte secretion of IL-6, to increase oligodendrocyte proliferation.

14. In the absence of microglia or astrocytes, $\mathrm{MBP}^{+}$oligodendrocytes had less branched processes and their number was reduced as well. Similar to findings on proliferation, the differentiation of oligodendrocyte progenitors was decreased by neutralization of IL-6, but not of CXCL1 secreted from astrocytes. We propose that a novel interaction between astrocytes and oligodendrocytes, the one that includes CXCL1/IL-6 signaling pathway, enhances development of human fetal oligodendrocytes.

15. During remyelination in multiple sclerosis, basic processes of oligodendrocyte development including proliferation, migration and differentiation of oligodendrocyte progenitors are recapitulated. For that reason it is of extreme importance to understand these processes during normal oligodendrogenesis. We describe here that constitutive expression of CXCL1 has physiological relevance, whereas exogenous CXCL1 might be relevant in pathological conditions, such as developmental disorders of white matter, or in multiple sclerosis.

16. A wide variety of signals that may be involved in initiation of myelination of CNS axons has been reported. Although mouse oligodendrocytes in vitro have the capacity to form myelin-like membranes in the absence of neurons, co-culture with neurons significantly increases MBP gene expression in these cultures, suggesting that axons are necessary for efficient myelination.

17. In vivo experiments in the rat optic nerve demonstrated that oligodendrocyte proliferation and subsequent myelination depend on the electrical activity in axons. Other studies have confirmed that ion channel activation or electrical activity in axons can regulate myelination, suggesting that oligodendrocytes preferentially myelinate axons which fire action potentials.

18. Myelination in the human brain progresses over several decades, which is much longer than a complete lifespan of the commonly studied animals. Recently emerged data suggest that myelination may play an important role in data processing by neurons. On the one hand, it is now clear that human white matter changes with experience, i.e. that myelinating glia respond to environmental cues, as shown by magnetic resonance imaging of neglected children. On the other hand, myelin thickness influences conduction velocity, which is in turn instrumental in regulation of synchronous firing of action potentials and proper function of the brain.

19. Several myelin proteins, including Nogo-A, MAG and MOG cause the growth-cone collapse that prevents axons from reaching their targets. This feature of myelin is now appreciated as an important regulatory mechanism to suppress sprouting and formation of abnormal connections in development.

20. Before myelination is initiated, oligodendrocyte precursors transform first to pre-myelinating oligodendrocytes and then into mature myelin-producing cells. During this process, myelin proteins, MBP and PLP, shift their expression from cell bodies to processes that form myelin sheaths. In humans there is a clear dissociation between the time of oligodendrocyte differentiation and the beginning of myelination in the fetal forebrain.

21. We demonstrated that a prominent increase in myelination coincided with the down-regulation of a polysialic acid conjugated form of the neural cell adhesion molecule (PSA-NCAM). This finding is consistent with the notion that a PSA-mediated signaling mechanism might be one of the regulators of primary myelination in the human fetal brain. 
It is important to stress that PSA-NCAM is not the only cell adhesion molecule to be implicated as an axonal inhibitor of primary myelination. Many other molecules, for example neural adhesion molecule L1, have been shown to be downregulated from the axonal surface upon the onset of myelination. This suggests that adhesion molecules are an important signal during the initial contact between axon and oligodendrocyte, but their downregulation is needed for myelination to proceed. Other types of molecules that are inhibitory for myelination comprise oligodendrocyte myelin proteins and their receptors.

22. Rodent vs. Human. Since most studies of mammalian brain development are done on rodents, we find it useful to understand how various time-points in rodent brain development correlate with much longer human brain development.

a. In the literature there are just a few attempts to relate processes during rodent brain development with those in humans. Previous attempt to draw parallels between development of experimental animals and humans resulted in designation of Carnegie stages. This classification is based on somatic morphology of embryos and it presumes that brain development is linearly predictable from somatic development, and that all brain regions develop at equivalent rates across species. Other attempts were done to make rat/primate comparisons based on neuroanatomy, comparing rat embryonic days 11-21 with human weeks 4-16. However, most of these comparisons do not take into account disparities in relative sizes of human brain regions.

b. Studies of human brain development from our laboratory suggest that processes like neurogenesis and oligodendrogliogenesis progress at a pace in the human brain, so every process has to be compared separately. Whereas neocortical neurogenesis does begin at $5 \mathrm{gw}$, corresponding to mouse embryonic day 10 , the ending of neurogenesis is not that clear, since at the last time-point of our study, 24gw neurons are still being born out of cells isolated from the cortical VZ/SVZ. Cortical oligodendrogenesis begins around $10 \mathrm{gw}$ in humans, but it progresses well into adulthood in humans. MBP expression peaks in the mouse cortical and subcortical white matter at P20, when primary myelination is completed.

c. Human primary forebrain myelination takes decades, compared to weeks in rodents. The most important reason for this prolonged development is that the much larger human brain has entire neocortical regions which rodent brains completely lack, whereas some of the key regions of rodent brains are in human relatively underdeveloped (e.g. olfactory bulbs).

d. Another interesting issue concerns the overall number of oligodendrocytes in human versus rodent brain. Although numbers of oligodendrocytes needed to myelinate large human brains are much higher than in rodents, it is not as clear if the densities and proportions of oligodendrocytes are different. In mouse cerebral and cerebellar cortex, for example, the density of oligodendrocytes was estimated to be $12.5 \times 10^{3} / \mathrm{mm}^{3}$, which makes for approximately $5 \%$ of all cells detected by the nuclear staining. Comparable stereologic studies of the human prefrontal cortex suggest very similar densities for oligodendroglia in humans.

\section{GENERAL COMMENTS AND DISCUSSION DeFELIPE}

Comment on point 23.3:

It is not clear for me what is the relationship between the prolonged time of myelination and the presence of more specialized neocortical regions in humans compare to rodents, and to the fact that some regions of rodent brains are in human relatively underdeveloped.

\section{ROCKLAND}

The theme of heterogeneity and cellular diversity re-appears, in the context of multiple generative sources of oligodendrocytes (point 7). As the authors comment, the implications of this fact are poorly understood, but could include important issues of species specialization (point 22).

\section{KOSTOVIC}

In this paper, correlation between oligodendrocyte development and other neurogeneic events is best documented for the subplate zone (remark 4). Dense accumulation of oligodendrocyte precursors in the zone of waiting thalamic afferents is important for later myelination of thalamo-cortical axons. Laminar specificity of glia distribution seams to be related to other neurogenetic events. Accordingly, oligodendrogliogenesis in the human brain is prolonged compared to rodent brain (remark 22).

\section{A commentary on}

Growth of the human corpus callosum: modular and laminar morphogenetic zones by Jovanov-Milosevic, N., Culjat, M., and Kostovic, I. (2009). Front. Neuroanat. 3:6. doi: 10.3389/neuro.05.006.2009.

\section{REMARKS AND MAIN CONCLUSIONS}

1. To accomplish complex tasks, mammals require coordinated brain activity, based on precise and efficient connections between the two hemispheres. These connections consist of axons that traverse the telencephalic midline, principally in three commissural tracts: the corpus callosum, the hippocampal commissure and the anterior commissure. Among these, the corpus callosum is the most voluminous fiber tract, and in the human species reaches its maximum complexity and size relative to brain volume.

2. Anatomical studies in experimental rodents demonstrated that the majority of contralaterally projecting (callosal) neurons are located in cortical layers II/III and layer V, while in the primate brain fibers of the corpus callosum predominantly originate from layer III pyramidal neurons of the neocortex.

3. Axons of the callosal neurons elongate to the intermediate zone, then navigate medially through a well-defined pathway along the medial wall of the ipsilateral ventricle, cross the midline, grow further into the contralateral hemisphere towards the target region and area (usually homotopic), and finally enter the appropriate cortical layer to establish functional connections. Members of the Netrin, 
Slit, Semaphorin, Ephrin and Wnt families of guidance molecules and their receptors, coordinate this extremely demanding navigation.

4. Several well-known developmental mechanisms, such as guidance by pioneering axons, guidance by pre-existing axonal tracts and guidance by cellular structures have been ascribed to various morphogenetic zones involved in the complicated pathfinding during the formation of commissures in a mammalian brain. However, these different morphogenetic zones have some principal properties in common: (1) strategic location (2) sequential appearance and dissolution, i.e. particular developmental window (3) modular or laminar appearance (4) versatile expression of guidance cues and (5) abundance of extracellular matrix (ECM). Disturbances in finely tuned expression of guidance and ECM molecules in the morphogenetic zones might cause structural or functional anomalies ranging from subtle cognitive impairment to severe developmental abnormalities, including dysgenesis and agenesis of the corpus callosum and other commissures.

5. As soon as they arise from the soma of future cortical neurons, the predetermined cortical efferents grow toward the intermediate zone guided by gradients of different guidance molecules. When they reach the intermediate zone, the axonal populations have to decide to grow medially around the ipsilateral ventricle as future callosal axons, or to grow laterally toward the internal capsule as long subcortically projecting axons, in both cases avoiding proliferative zones which express repelling cues, e.g., semaphorin3A (SEMA3A). Data regarding this decision point in human callosal formation are still missing, but exuberant axonal bifurcations have been reported in mice.

6. The early growth of future callosal axons in humans and the subsequent morphogenesis of the callosum were firstly demonstrated by histological methods and described in the classical embryological studies. Prior to corpus callosum formation, at 11 postconceptional weeks (PCW), a new structure designated as massa commissuralis is rapidly formed after the fusion of median groove banks above the septal area in the so called "commissural plate of Hochstetter". The first axons, named pioneering, approach and penetrate the massa commissuralis at the mediosagittal plane after $11 \mathrm{PCW}$. Therefore, the medially positioned massa commisuralis is probably the first midline structure that expresses specific molecules and morphogenes for guidance and nurture of pioneering callosal axons in humans.

7. Correlation of in vitro MRI and histological analysis of the developing human cerebrum revealed that the commissural plate, as well as other transient fetal zones, can be visualized on T1-weighted images already at 10 PCW. The commissural plate is then at the onset of its development and visibility on MRI scans as a thickened dorsal part of the telencephalon impar. The callosal fibers that penetrate massa commissuralis and form the callosal plate, can be demonstrated by histological methods at 12-13 PCW, while the earliest stage of their visualization by DTI is at $14-15$
PCW. Some reports have suggested that the first axons are the ones that will form the rostrum, the genu and the body, while others suggest that the callosum will grow in both anterior and posterior directions, with a more prominent anterior growth.

8. With the formation of the callosal plate, several morphogenetic zones appear along the midline and subsequently develop into transient cellular structures: the midline sling, the glial wedge and the glia of indusium griseum (indusial glia). These developmental structures were first recognized and described in morphological studies of experimental animal models. Later they were further explored in mice by modern molecular methods, and for each structure a critical role in the morphogenesis of the corpus callosum has been established. Recently, histological and correlated histological/MRI studies demonstrated that these transient structures are also present during the period of early development of the human forebrain midline, indicating conservation of developmental mechanisms and structures during mammalian evolution. These morphogenetic structures situated in strategic locations have overlapping developmental windows between 13 and 20 PCW.

9. The midline sling was originally described as a thin concave lamina consisting of migrating glia-like cells that tightly underline the ventral surface of the developing corpus callosum. It was later proven that, at least in mice, a substantial portion of cells that forms the sling are neurons. Neuronal nuclear antigen (NeuN), CR and glial fibrillary acidic protein (GFAP) have been demonstrated in the human midline sling, indicating that it also consists of neuronal and glial cells. The origin of the cells that compose the midline sling in humans is complex and not clearly understood.

10. The list of guidance molecules, transcription factors and morphogens involved in corpus callosum formation in mice and humans, which are expressed either in the midline structures or by growing callosal axons, is continuously expanding. However, evidence for expression of these molecules in the human brain midline is still very scanty.

11. Studies in experimental rodents have demonstrated that ECM molecules such as laminin, fibronectin, proteoglycan NG2, heparan and chondroitin sulfate proteoglycans play an important role in the guidance of commissural axons. So far, in the human brain only tenascin- $\mathrm{C}$ was convincingly shown to be present dorsally and ventrally to the corpus callosum until 20 PCW. ECM molecules rather than just constituting a structural scaffold, interact with guidance cues and can critically modulate axonal guidance function.

12. Taken together, studies on human brain development have confirmed that developmental mechanisms governing the early formation of the forebrain midline and corpus callosum are indeed very similar to, if not the same as those described in experimental rodents. However, the human fetal brain is significantly larger than the brain of prenatal rodents and the distances the growing commissural axons have to cover are notably longer in every segment of their complex journey. Therefore, it is reasonable that commissural connections in 
humans require a longer period of contemporaneous persistence of supporting midline structures and probably additional morphogenetic zones.

13. There are only a few histological studies describing the later stages of corpus callosum development in humans. In fetuses at $18-20 \mathrm{PCW}$, it is a well-developed fibrillar structure, which in coronal planes, runs transversally toward the midline and after crossing curves along the roof and lateral wall of the lateral cerebral ventricle. In the midsagittal plane, the outlines of the major callosal parts (genu, body and splenium) are clearly visible and assume the same shape and position as in the adult brain, with the exception of being much smaller in their rostrocaudal extent and thickness. DTI studies of the fetal brain at 19 PCW show the callosal radiation with its typical shape, similar to the one seen in neonates, with axons from all parts of the callosum forming a mohawk-shaped structure. At this stage the callosal cross-sectional area is only $5 \%$ of the size seen in a 5-year-old infant. At the neonatal stage, just before apparent myelinization of the callosum starts, it is half the size.

14. Beside the early midline structures, a morphogenetic role in formation of the human corpus callosum during the second half of gestation has been described for two additional zones: callosal septa and subcallosal zone (SCZ). These two midline structures jointly form "grooves" in which callosal bundles are laid down during the second half of gestation.

15. Postmortem analysis of the corpus callosum (age range from the 18 PCW to adult), immunostained for GFAP, NeuN and chondroitin sulfate proteoglycan (CS-56), in addition to classical histological methods, revealed the existence of modular cellular structures; namely, the callosal septa, which are most prominent during the second half of gestation.

16. During the developmental window of 18-34 PCW, the number of callosal septa is individually variable. Usually 15-20 thicker and longer septa and numerous smaller septa are unevenly distributed along the anteroposterior axis. In the genu and the anterior part of the callosal body, the septa are more numerous and more regularly spaced. In the rest of the callosal body, their number declines, while in the splenium it increases again, but the septa are still less numerous and less prominent there, in comparison with the anterior portion of the callosum. At the cellular level, the callosal septa contain: GFAP reactive meshwork, NeuN positive neurons, CS-56 immunoreactive ECM and expression of the guidance molecule SEMA3A in cells and ECM. At midgestation, SEMA3A is expressed in septa of the anterior third of the callosum and above the fornix. It is important to note that chondroitin sulfate proteoglycan in the ECM plays an important role in the cellular localization of SEMA3A, and their interaction can modulate the biological activity of this guidance molecule.

17. The callosal septa have not been shown by conventional MRI, yet the abundance of ECM and proteoglycans in septa, as well as their radial orientation between callosal bundles, should influence the MRI signal in diffusion-weighted imaging of the human fetal brain.

18. The ventral part of the callosal septa is in continuation with the SCZ, which like a thin lamina occupies the median and paramedian territories situated between the developing cor- pus callosum (dorsally) and the fornix bundles (ventrally). The cellular composition of the SCZ (revealed by acetylcholinesterase histochemistry and Golgi staining) is characterized by area-specific neuron-like cells with long and wavy processes, large glia-like cells, maturing neurons, migratory-like neurons and radial glial cells, with a difference in distribution between the medial (nucleus septohippocampalis) and lateral portion (allocortical counterpart of the SVZ).

19. During the early postnatal period, the callosal septa become thinner and shorter, lose their neuronal and chondroitin sulfate proteoglycan content. At the same time, the number of cells in the SCZ decreases.

20. The corpus callosum of adult primates consists of "segments" which contain topographically segregated callosal fibers for a given cortical area. Thus, callosal septa with their topographic arrangement, shape and content most likely provide a basis for topographically ordered commissural projections from one hemisphere to another, or at least maintain the topographical relationship during development. Confirmation of this suggestion can be found in the fact that the basic topography and terminal field patterns of callosal projections in monkey brain are established already by E133, well before birth.

21. In addition, tractography studies in humans showed an evident dorsoventral fiber distribution in the adult human brain, with earlier developed medial cortical areas sending their fibers dorsally through the corpus callosum, while later developed laterodorsal cortical regions send them ventrally. In this respect, callosal septa and SCZ with their abundance of ECM and guidance molecules along the ventral aspect of the corpus callosum are strategically located for influencing the growth of callosal axons.

22. One of the principal mechanisms of cortical development is an overproduction of axons, axonal branches and synapses, the so-called developmental exuberance, which is followed by a subsequent selection and refinement based on adequate target region recognition and activity of the functional connections. The corpus callosum is a pivotal example for such developmental exuberance, since the number of callosal axons in monkeys at E165, exceeds the number of callosal axons present in the adult by at least 3.5 times. Thus, the later morphogenesis of the corpus callosum is even more complex due to the processes of retraction of exuberant callosal fibers. In the primate brain, this continues during the first three postnatal months. In the human brain, decrease in size of midsagittal cross-sectional area was observed after $32 \mathrm{PCW}$ and this lasts to the second postnatal month. This suggests the beginning of a retraction of exuberant callosal axons, concomitantly with the resolution of waiting compartments and the resolution of the majority of the callosal septa. The remnants of septa that continue for some time after birth may help in the process of withdrawal of exuberant callosal axons, since these structures and processes temporally overlap.

23. In addition, the septa may also provide corridors for migration of later-born neurons, since thick callosal bundles on the roof of the lateral ventricle represent a structural barrier. The 
groups of neurons originating from the SVZ may use glial fibers in the callosal septa to "climb", while guidance molecules and ECM facilitate migration towards the cortex.

24. Concluding remarks.

a. Recent progress in gene targeting methods, advances in axonal tracing, and high-resolution MRI techniques have revealed the morphogenetic zones and their role in guiding callosal axons across the midline.

b. Although, the corpus callosum at midgestation assumes a shape and position not essentially different from that in the adult brain, it is still far from its definitive rostrocaudal extent and thickness.

c. In the developing brain, all histogenetic events (neurogenesis, gliogenesis, migration, cell differentiation, axonal extension and synaptogenesis) proceed within laminar or modular compartments or zones. These do not have an equivalent in the adult brain. Therefore, different structures and processes have to be interpreted in a precisely defined spatial and temporal context. In that respect, it is important to note that in humans the complex event of interhemispheric integration through the corpus callosum continues throughout gestation and well after birth. The second half of gestation and the early neonatal period are important for the multiple-fold increase of callosal axonal number, selection of functional axons, withdrawal of exuberant axons and targeting of the region, area, layer and cells of the cortical plate.

d. Insight into the organization of the morphogenetic zones involved in development of cortico-cortical pathways is a prerequisite for the studies of preterm and term-born infants in normal development and pathological conditions.

e. Considering the clinical significance of the corpus callosum (over 50 different syndromes display dysgenesis of the corpus callosum and over 40 genes are linked to these anomalies, its importance for cognitive functions and its frequent lesioning in the perinatal period, we would strongly encourage long-term studies of the formation of commissural pathways in humans.

\section{GENERAL COMMENTS AND DISCUSSION DeFELIPE}

General comment:

I was wandering if there are gender differences in the growth of the human corpus callosum.

\section{ROCKLAND}

I recently learned that agenesis of the corpus callosum is accompanied by vertical fingerlike structures (Probst's bundles) in the interhemispheric walls. Can the authors (or Others) comment on a possible relation to the developmentally important septa?

\section{ROCKLAND}

Comment on point 2:

I would say that callosal connections between the association areas, in particular, often involve layer 5 as well as 3 , in macaque. This raises another possibly useful species difference, as I believe callosal connections in rodents originate more equally from layers 3 and 5 ?

\section{A commentary on}

Could sex differences in white matter be explained by g ratio? by Paus, T., and Toro, R. (2009). Front. Neuroanat. 3:14. doi: 10.3389/ neuro.05.014.2009.

\section{REMARKS AND MAIN CONCLUSIONS}

1. In vivo studies of the human brain have revealed the presence of striking sex differences in the volume and microstructure of white matter. In adults, the overall volume of white matter (WM) is higher in men than women; in most studies, this is true even after accounting for sex differences in brain size. On the other hand, sex differences in the "density" (the term "density" or "concentration" refers to the probability of classifying a voxel as belonging to a specific type of tissue without making any inference about possible underlying neurobiological processes) and FA of WM constituting various fibre tracts appear to vary across the tracts, being higher in women than men in some WM pathways and vice versa in others.

2. Many of these sex differences in WM emerge during adolescence. We and others have shown a steep increase in the WM volume during adolescence in boys but not girls.

3. The total volume of white matter is determined by the number of axons, their calibre and the thickness of myelin sheath produced by the oligodendrocytes. Given the known elimination of axons in the early post-natal period, age-related increases in the volume of white matter during brain development in childhood and adolescence can be accounted for by increases in axonal calibre and/or thickness of the myelin sheath.

In the same sample of typically developing adolescents, we have observed the following three phenomena: (1) WM volume increases steeply during adolescence in boys but not girls; (2) magnetization-transfer ratio (MTR) decreases with age in boys while it does not change (or increases slightly) in girls; and (3) WM density in the cortico-spinal tract (CST) decreases with age in adolescent boys but increases in girls. We have proposed that these phenomena may be best explained by a disproportionate growth of the axon compared with the growth of its myelin sheath and, as such, by changes in $g$ ratio (axon diameter/fibre diameter; see below). We have speculated that a subtle shift in g ratio in WM of the male brain during adolescence would affect the proportion of WM tissue occupied by axons and myelin, thus explaining the emergence of sex differences in WM density and MTR described above.

4. G ratio refers to the ratio between axon diameter $\mathrm{d}$ and fibre diameter D; fibre diameter is the sum of the axon diameter (or calibre) and the thickness of the myelin sheath.

5. In the monkey corpus callosum (CC), unmyelinated axons, accounting for about $30 \%$ of all axons in the genu and less than $10 \%$ elsewhere in the CC, have a small diameter $(\sim 0.1 \mu \mathrm{m})$, whereas the diameter of myelinated axons varies between 0.1 and $2.5 \mu \mathrm{m}$, with the largest axons $(2.5 \mu \mathrm{m})$ found in the CC midbody. The largest axons in the human brain are found in the internal capsule and, most likely, correspond to the cortico-spinal neurons; the size of these axons 
increases from birth $(\sim 1 \mu \mathrm{m})$, through childhood $(12 \mu \mathrm{m}$ at 7 years of age) into adulthood $(24 \mu \mathrm{m})$. These numbers correspond to the fibre diameter and, as such, reflect both the radial growth of the cortico-spinal axons and their increased myelination with age. We have suggested that changes in the WM density in the corticospinal tract observed in our studies in relation to plasma levels of testosterone reflect changes in the diameter of these axons.

6. In general, axons of large diameter have a thick myelin sheath. It turns out that the $g$ ratio is relatively stable at $\sim 0.6$. This observation, together with the small diameter of unmyelinated axons, suggests possible coupling between myelination and axon diameter. But the range of $g$ ratio varies considerably across axons of the same fibre tract, as a function of age, mouse strain or disease.

7. Importantly, g ratio appears to increase as a function of axon diameter, indicating a relatively thinner myelin sheath in large axons.

8. By assuming that geometrically similar fibres should possess similar membrane properties, Rushton derived a formula showing that, for a constant $\mathrm{g}$ ratio, the conduction velocity $\mathrm{v}$ and fibre length 1 should be proportional to the fibre diameter.

9. What determines axonal diameter? Axon consists mainly of neurofilaments (NF) and microtubules (MT), the former outnumbering the latter 5-10 times. Neurofilaments support the cylindrical structure of an axon and, as such, protect the core from compressive stress, securing its unobstructed state. Axonal diameter is influenced both by the number of NF and their spacing. The former is regulated by NF synthesis (gene expression) and the amount of NF undergoing (slow) axonal transport; not surprisingly, the number of NF, but also that of MT, varies as a function of axon diameter. The latter depends on the phosphorylation of NF side arms. This process appears to be regulated by a protein synthesized by oligodendrocytes, namely myelin-associated glycoprotein; this "outside-in" signalling pathway provides a cellular mechanism for the coupling between myelination and axonal calibre. Theoretically, sex differences in $g$ ratio could emerge either through differences in the rate of synthesis and/or axonal transport of NF, or the rate of phosphorylation of the NF side arms.

10. Conclusions and implications.

a. The initial work from our laboratory has suggested a disproportionate growth of axon and myelin sheath, respectively, during male adolescence. This phenomenon turned our attention to possible sex differences in $g$ ratio. Both the experimental work reviewed above and the model predict lower concentration of myelin in WM regions occupied by large-diameter fibres; we have observed, indirectly, this phenomenon in the cortico-spinal tract at the level of internal capsule. It is likely that the male brain contains, overall, a larger proportion of largediameter fibres. What might be the neurobiology underlying such a sex difference?

b. Although the number of MT also increases with the axonal diameter, directionality of this relationship is not clear. The link between testosterone and cellular processes relevant vis-à-vis cytoskeleton are rather tentative at present. Initial in vitro studies suggest that testosterone up-regulates $\alpha$-and $\beta$-tubulin, the building blocks of microtubules. Furthermore, neuropathy is one of the key symptoms of Kennedy's disease (or X-linked spinal and bulbar muscular atrophy), a condition caused by an expanded polyglutamine (polyQ) stretch in the androgen receptor. This effect might be mediated by a PolyQ-AR induced inhibition of kinesin-mediated axonal transport or a decrease in the expression of dynactin 1, a motor for retrograde axonal transport; slow axonal transport is essential for moving elements of cytoskeleton.

c. Is the rate of axonal transport different in small-and largediameter fibres? This appears to be the case. Murayama and coworkers used MRI to measured the rate of in vivo transport of $\mathrm{Mn}^{2+}$ ions from the eye to the lateral geniculate nucleus of the monkey and found that a faster transport in the magno-cellular that parvocellular pathway. The $\mathrm{Mn}^{2+}$ transport depends, at least in part, on kinesinbased processes underlying (active) axonal transport.

d. In general, axonal cytoskeleton (i.e., NF and MT) and motor proteins are essential contributors to a large number of cellular processes, such as cell metabolism (e.g. transport of mitochondria and glycolytic enzymes) and neurotransmission (e.g. transport of synaptic-vesicle precursors), as well as growth and cell survival (e.g. retro-grade transport of growth factors). Thus, any sex differences in axonal transport may have a number of downstream effects.

e. The model presented in this article suggests that the $g$ ratio will increase in large fibres beyond the optimal value of 0.6. We speculate that the presumed testosterone induced changes in axonal diameter during male adolescence increase the probability of suboptimal g ratio in largediameter fibres and, in turn, decrease conduction velocity in these fibres. Such a disturbance may interfere with the timing of inter-regional communication and contribute to the emergence of a "disconnection" syndrome hypothesized to underlie disorders such as schizophrenia.

\section{GENERAL COMMENTS AND DISCUSSION DeFELIPE}

Comment on point 3:

The authors claim that the total volume of white matter is determined by the number of axons, their calibre and the thickness of myelin sheath. However numerous neurons are present in the white matter. Do the authors think that the presence of these neurons has little impact on the volume of white matter?

\section{HÖISTAD AND HOF}

Paus and Toro observed differential increases in WM volume in boys compared to girls during adolescence (i.e., after 12 years of age). They propose that there is a disproportionate growth of axons compared to the growth of myelin sheaths during male adolescence (Perrin et al., 2008). It remains to be determined whether, or how, sex hormones play a role in this development.

Inherent to the issues of axon and myelin growth, is the issue of identifying a possible coupling between myelination and axon diameter. For example, several thin axons are unmyelinated, whereas 
large diameter axons are heavily myelinated. As such, what determines axon diameter, and what are the signals required for the axon to get myelinated? Paus and Toro discuss the link between axon diameter and neurofilament, the main axonal cytoskeletal protein. It turns out that neurofilament phosphorylation, which determines neurofilament spacing, may in fact be regulated by MAG (myelin associated glycoprotein) expressed in oligodendrocytes (Yin et al., 1998). These authors have shown that MAG modulates caliber, neurofilament spacing, and neurofilament phosphorylation of myelinated axons, and that absence of MAG results in axonal atrophy and Wallerian degeneration of myelinated fibers. Thus, MAG may provide one of the mechanisms that are responsible for the coupling between myelination and axonal caliber. Interestingly, it has been found by Hof and coworkers that MAG deficient mice do not exhibit any changes in FA of the cingulum bundle as measured by DTI, nor do they exhibit any changes in fiber length densities of the cingulum bundle as assessed by stereologic analysis (Segal, 2008). This may point to the fact that not all pathways are equally affected in the MAG knockout mouse. It is equally possible that sex hormones exert a direct or indirect effect on myelination or axon growth through mechanisms unknown as yet, which represent interesting targets for investigations considering the gender-dependent differences in white matter that occur during adolescence.

\section{A commentary on}

Myelination and isochronicity in neural networks by Kimura, F., and Itami, C. (2009). Front. Neuroanat. 3:12. doi: 10.3389/ neuro.05.012.2009.

\section{REMARKS AND MAIN CONCLUSIONS}

1. The timing when a neuron receives its incoming input has a great influence on how the input is processed and how it affects the postsynaptic neurons. On the other hand, an increase in the body size and the amount of information processed, as a result of evolution, inevitably required the expansion of the brain. This resulted in situations where the excitation of a presynaptic neuron needs to arrive within a fixed window of time at target neurons located at multiple remote sites at variable distances. Such apparently paradoxical transmissions, in fact, take place in various regions of the brain. Some representative examples of such isochronicity can be observed in olivocerebellar connections, transcallosal connections in the visual cortex and amygdalo-cortical pathways.

2. Intriguingly enough, these connections do not necessarily adopt the same strategy to accomplish isochronicity. Recently, we found that the thalamocortical pathway also exhibits the isochronic property, but with a novel mechanism, which involves differential myelination along the axon. Apparently this is an exquisite way of producing isochronicity and may apply to other systems.

3. Neurons in the thalamus send axons to a wide area of the somatosensory cortex through different trajectories of various travelling lengths. Nevertheless, we reported that action potentials in the thalamic cells arrive almost simultaneously at each target cortical cell in layer IV. We found that the isochronicity in this system is achieved by changing the conduction velocity (CV) within the individual axons. The CV decreases significantly upon entering the gray matter by up to 10 -fold. Originating from the thalamus, the axons of relay cells run in a straight path through the striatum up to the WM, at which point each axon diverges widely. Some ascend directly into the cortex up to layer IV, while others run in the subcortical WM for variable distances before ascending into the cortex. Although the total distances travelled vary across pathways, the distance of the intracortical regions is almost the same. That is, all the projections target the same layer IV in a cortex that has uniform thickness. The total conduction time primarily depends on the time spent within the cortex (gray matter). Thus, the strategy of making the CV of longer and variable parts by far faster than that of shorter and constant parts is a way of eliminating the variability in traversed distances.

4. Myelination is involved in this process in two-fold. First, since $\mathrm{CV}$ is proportional to the square root of the axon diameter in unmyelinated axons, to have 10 times larger CV, axon diameter needs to be 100 times larger, which is most unlikely. This led us to predict that thalamocortical fibers would be myelinated. Thus, secondly, we suspected that the extent of myelin might cause the observed difference of CV. Histological staining of the myelin revealed that the difference of myelination between the intracortical and extracortical (WM) regions played an important role in the generation of $\mathrm{CV}$ difference.

5. Isochronic property of transcallosal connections is one of the most intensively studied within the framework of the computational properties of axons by Innocenti and coworkers. Based on the simulation of action potential propagation determined by axonal diameter, these authors provided a detailed description of strategies for callosal connections to achieve synchronous activation of their targets.

6. In the cortex, layer $\mathrm{V}$ pyramidal neurons project to various subcortical regions as well as to the contralateral side. Chomiak and coworkers showed that layer V pyramidal neurons in the ventral temporal lobe innervate diverse regions such as the caudate putamen, parietal cortex, amygdala, and thalamic nuclei on the ipsilateral side with isochronic spike delivery based on the differential CVs in each fiber branch. By combining the actual measurement of the axonal inner and outer diameters, with theoretical predictions based on partial myelination, the observation of Chomiak and coworkers on isochronicity appears to be best supported by partial or differential myelination. It may be worth mentioning that the same neurons send axons to the contralateral side through the corpus callosum, but the CVs were significantly slow and not isochronic with ipsilateral connections.

7. One implication from these studies reviewed here is that each pathway has its own conduction time, which might be determined by its function. Myelination, axon diameter, and other factors such as the structure, or the number of spacing of Ranvier nodes, and ion channel composition and/or its density, all of these would affect the $\mathrm{CV}$, and conduction time may be employed to achieve its characteristic value. On the other hand, since the environment around a given neuron, or 
tissue, or whole individual continuously changes, a regulatory mechanism that changes in an input- or activity-dependent manner is desirable to adapt to the changing environment.

8. Myelination indeed changes in an experience- or inputdependent manner. For example, the DTI. study of Bengtsson and coworkers revealed that extensive piano practicing in childhood results in a thicker white matter, which is believed to be due to a change in myelination.

9. Several lines of evidence have identified how action potentials regulate myelination. A specific pattern of neural activity was shown to lower the expression of L1-CAM that is necessary for myelin induction by oligodendrocytes, as well as by Schwann cells. In addition, ATP released from axon terminals as a result of neural activities facilitates the differentiation of oligodendrocytes through adenosine and P1 receptors. For astrocytes, ATP causes the release of leukemia inhibitory factor (LIF), which then stimulates myelination by oligodendrocytes.

10. The corpus callosum displays a significant variety in terms of myelination. For example, in humans, none of the callosal fibers are myelinated at birth, and in adults $30 \%$ of the fibers remain unmyelinated. In addition, analyses of fiber composition revealed a wide variety of fiber diameters and extent of myelination depending on the target area. Callosal regions connecting prefrontal and temporoparietal association areas consist of small caliber with low myelinated fibers, whereas regions connecting primary and secondary sensorimotor areas include highly myelinated, large-caliber fibers. Consequently, the conduction time between two hemispheres varies from $30 \mathrm{~ms}$ via myelinated axons to as long as $300 \mathrm{~ms}$ via unmyelinated ones. Since callosal fibers connect a variety of cortical regions with various functions, conduction times for each functions are likely to be diverse. The extent of myelination, as well as axon diameter might help regulate the conduction time to its optimal value for communicating between hemispheres. This leads to another interesting question; namely, how the conduction time of each pathway is determined in the network.

\section{GENERAL COMMENTS AND DISCUSSION DeFELIPE}

Comment on points 7 and 10 :

If each pathway has its own conduction time and isochronous activation of thalamocortical and cortico-cortical connections are thought to be crucial in the binding mechanisms of sensory perception, how can both arguments could be related to explain the global binding?

\section{ROCKLAND}

Concerning the statement of differential myelination along the axon (point 2), light microscopic analyses of individual axons have remarked what appears to be changes in axon caliber (Innocenti et al., 1994; Rockland and Drash, 1996). Since it has been difficult to investigate identified axons along their full length, data on this point are largely lacking. However, the changes and specializations along the trajectory of a single axon are likely to be a source of interesting and potentially important future research.

\section{A commentary on}

Linking white and grey matter in schizophrenia: Oligodendrocyte and neuron pathology in the prefrontal cortex by Höistad, M., Segal, D., Takahashi, N., Sakurai, T., Buxbaum, J. D., and Hof P. R. (2009). Front. Neuroanat. 3:9. doi: 10.3389/neuro.05.009.2009.

\section{REMARKS AND MAIN CONCLUSIONS}

1. Most neurons in the brain necessitate adequate myelination of their axons in order to maintain functional processing at all levels of neural systems, from autonomic processes and sensorimotor integration, to mood and thought.

2. The importance of myelination for cognitive functioning becomes apparent in diseases that are known to be caused or affected by deficiencies in myelin, where patients show deficits in intellectual, social and emotional functioning.

3. Leukodystrophies and leukoencephalopathies, diseases characterized by progressive degeneration of the white matter, if diagnosed in late adolescence or early adulthood can present with psychotic symptoms sometimes indistinguishable from those of schizophrenia. Likewise, patients with multiple sclerosis who display cognitive and psychiatric symptoms frequently have white matter lesions in the frontal and temporal lobes, which are the brain regions most implicated in schizophrenia.

4. Schizophrenia is a severe psychiatric illness that affects close to $1 \%$ of the population worldwide. The diagnosis is generally established at first onset of the symptoms, which occurs in most cases in early adulthood. The disease is characterized by a number of mental abnormalities that result in a distortion of perception and expression of reality. There are prominent sensory symptoms, most frequently taking the form of auditory and visual hallucinations, although such sensations can affect any sensory modality. In addition to hallucinations, the patients may experience paranoid delusions, present with disorganized thoughts and speech, and a variable degree of social and occupational dysfunction. There is a considerable degree of inheritability of the disease and prenatal causes, such as insult to the brain during embryonic development, have also been considered to play a key role in the expression of the disease at a later time.

5. Schizophrenia has been shown to exhibit myelin deficiencies and changes in white matter volume in the brain. The myelin hypothesis in schizophrenia was first presented by Hakak and coworkers after their pivotal finding of altered expression of myelin-related genes in human postmortem tissue. Myelinrelated gene expression levels have matched the observations made on white matter abnormalities by DTI, and were later confirmed in several other studies. Since the first suggestions of a myelin-related pathophysiology underlying schizophrenia, there have been numerous and extensive reports and reviews on the myelin hypothesis.

6. Substantial deficits in myelination occur in schizophrenia, which is interesting to consider in light of previous hypotheses that the disease results from abnormal brain development and altered neuronal circuitry, particularly in the prefrontal cortex (PFC). Neuropathologic findings in both white matter 
and grey matter suggest that myelin alterations in the anterior cingulate cortex (ACC) may underlie some of the behavioral deficits related to prefrontal dysfunction.

7. Fractional anisotropy (FA), a measure of the directionality of water movement within the spaces in-between axons, provides an indication of white matter tract directionality and, by measuring the strength of the direction vector of water diffusion, possibly of tract integrity or coherence. A major advantage of this approach is that it can be used to study changes in schizophrenia in vivo, allowing investigation of different stages of the disease. In vivo DTI studies have revealed decreased FA in several major white matter tracts in schizophrenia, including the cingulum. In addition, positron emission tomography (PET) imaging has demonstrated increased relative metabolic rates in white matter in schizophrenia, which may represent white matter inefficiency or defects resulting in increased metabolic needs, in contrast to findings in the grey matter which have shown decreases in regional cerebral blood flow in the ACC.

8. Although previous DTI studies have shown decreases in FA in the cingulum bundle as well as in the overlying cingulate gyrus in patients with schizophrenia, the findings have been somewhat inconsistent, due in large part to small subject samples and different methods of identifying particular brain regions of interest.

9. MRI studies of the grey matter have revealed regionally reduced cortical volumes in schizophrenia, including the ACC

10. In attempts to localize and identify a cellular correlate of the white matter changes observed by brain imaging in vivo, oligodendrocytes have come to be an important focus of investigation. Analyses of the number, densities and distribution patterns of oligodendrocytes can be performed in both the white and grey matter. Stark and coworkers found decreased oligodendrocyte densities in cingulate area 24 but not in the adjacent paracingulate area 32, and Hof and coworkers found decreased densities of oligodendrocytes in the prefrontal area 9 of the superior frontal gyrus in subjects with schizophrenia. In contrast, in a subsequent study, Hof and coworkers evaluated the degree of oligodendrocyte clustering in the anterior cingulum bundle, but found no differences using postmortem tissue from chronic schizophrenics versus age-matched controls. On the ultrastructural level, electron microscopy studies of oligodendrocytes in the PFC have demonstrated apoptotic oligodendrocytes, irregularities of mitochondria in oligodendrocytes and damaged myelin in area 10 in schizophrenic brains.

11. Postmortem studies, assessing the gyrification index, have found reductions in cortical folding in schizophrenia. Studies on changes in neuronal densities in different cortical regions in schizophrenia have been conflicting, and no definite pattern of neuronal density alterations has yet been established.

12. Cytoarchitectural studies have analyzed neuronal arrangements in terms of interneuronal distances, or mean cell spacing showing that mean cell spacing was reduced in area 9 in schizophrenic patients, which would imply a higher neuronal density. Rajkowska and coworkers found that in area 9, there was a downward shift in neuronal sizes, accompanied by increases in the density of "small neurons" in layer II, interpreted as GABAergic interneurons, while there was a decrease in the density of "very large neurons" in layer III, presumably pyramidal neurons, in patients with schizophrenia. Concomitant morphological studies at the single neuron level have demonstrated impoverished dendritic structures of pyramidal neuronsand loss of dendritic spines in schizophrenia, as well as in non-human primate models.

13. Another interesting finding is an anomalous distribution of the so-called interstitial white matter neurons in schizophrenia. These interstitial neurons have been suggested to be remnants of subplate neurons that normally undergo programmed cell death during brain maturation. However, in certain species including human, these white matter interstitial neurons are to some degree normally found in healthy adults. The interstitial white matter neurons have been found to be increased in prefrontal white matter and temporal white matter in subjects with schizophrenia, supporting further the presence of a neurodevelopmental abnormality in schizophrenia.

14. It is possible that the regionally specific remodeling of grey and white matter that takes place into the third decade of life underlies some of the structural and functional changes that leads to the development of psychiatric disorders such as schizophrenia. The fact that the PFC matures last and that myelination is not complete until late adolescence may be significant, as the timing coincides with the typical onset of symptoms in schizophrenia. This suggests that a dysfunctional myelination process could underlie the pathogenesis of schizophrenia. However, it is interesting to note the lack of neurological comorbidities in schizophrenia in comparison with other more typical white matter diseases. In dysmyelinating and hypomyelinating diseases such as the leukoencephalopathies, the effects of a myelin deficiency may be striking and fatal.

15. If the myelin hypothesis holds true, and myelin deficiencies prove to be one of the central causes of the development of schizophrenia, one might argue and question why classic schizophrenia patients show so few neurologic symptoms. Several other white matter abnormalities often generate disturbances at the neuron level, such as seizures and/or psychomotor developmental delays. Why patients with schizophrenia do not particularly exhibit similar neurologic co-morbidities, such as seizures or sensorimotor deficits, is unknown. It may be that only specific pathways become myelin-deficient, such as the late developing and poorly myelinated regions of the PFC, leading to the generation of behavioral symptoms seen in schizophrenia. Since the diverse circuits in the brain do not mature at the same time, if there is a developmental insult, this may affect only a certain population of neurons undergoing myelination, and result in a pathway-specific deficiency.

16. In a groundbreaking study using gene microarray analysis to examine gene expression levels in postmortem samples from schizophrenia patients Hakak and coworkers found that the expression of six myelin-related genes predominantly expressed in oligodendrocytes, was significantly decreased in the DLPFC in postmortem schizophrenic brains. The decreased expression of oligodendrocyte-related gene products was later confirmed and extended to other brain areas, implying that there 
is a pathology of oligodendrocytes underlying schizophrenia. Genetic linkage studies have also implicated myelin-related loci in schizophrenia although linkage studies are now considered somewhat controversial in complex psychiatric disorders.

17. Much evidence, including whole genome association studies, have identified myelin- and oligodendrocyte-related genes as susceptibility genes for schizophrenia. One of the most promising schizophrenia-related genes is neuregulin 1 (NRG1) gene. NRG1 and the NRG1-receptor ERBB4 are involved in several aspects of nervous system development including oligodendrocyte development Given the emerging role of NRG1 and ERRB4 in oligodendrocyte development, it is possible that alterations in NRG1 and ERBB4 affect oligodendrocytes, leading to schizophrenia.

18. Transgenic mouse models may serve as vehicles for studying the morphological and anatomical abnormalities that may result from a genetic defect affecting myelination. Some recent mouse models of white matter dysfunction have emerged during the last few years, which may serve as putative animal models for schizophrenia.

19. Evidence from very different lines of research supports the premise that dysfunction of oligodendrocytes is a critical factor in the development of schizophrenia. The precise role oligodendrocytes hold in the cascade of malfunctions that results in the constellation of deficits seen in the disease is still unknown.

20. Future directions

a. Layers II and III pyramidal neurons in the ACC may be the targets of axonal pathways affected in schizophrenia. Quantitative information on neuronal integrity in mouse models is important for understanding downstream effects of myelin genetic abnormalities, and to assess the validity of models in the context of observable neuropathologic changes in human brains. These studies need to be extended to additional models reflecting the genetic complexity of schizophrenia, and electron microscopy studies should be used further to assess structural aberrations in oligodendrocytes and myelin sheaths, as well as immunogold approaches to study synaptic integrity by visualizing preand postsynaptic proteins.

b. Correlative morphology and density analyses of dendritic spines will help clarify plastic changes in responses to myelin challenges. The data obtained in transgenic mice will offer critical correlates to neuropathologic features that can be analyzed in postmortem human materials. Combined analysis of human specimen and relevant mouse models offers a unique opportunity to investigate myelin deficits that have a clinical impact. As a result of such combined approaches, a model of schizophrenia with characterized molecular defects that can be used for developing therapeutic approaches will hopefully emerge.

\section{GENERAL COMMENTS AND DISCUSSION \\ DeFELIPE}

General comment:

The authors state that schizophrenia has been proposed to arise partly from altered brain connectivity. Could the authors, or whoever is interested, comment from his/her own experience on what particular connections are affected? How specific are these changes? Are GABAergic neurons affected or is it thought that mostly pyramidal neurons are vulnerable?

\section{ROCKLAND'S}

Response to DeFelipe's comment:

To the extent that long-distance connections are involved, this will be hard to determine in humans (as long as DiI or comparable post-mortem tracer remains refractory.) Also, it is hard to know what level to search: presynaptic arbors or synapses? Postsynaptic targets? Receptors, so this will involve a concerted ongoing effort. One related recent report is Rinaldi et al. (2008).

\section{DeFELIPE}

Comment on point 20.1:

It is not clear to me why only layers II and III pyramidal neurons in the ACC may be the targets of axonal pathways affected in schizophrenia. Are not affected pyramidal neurons in other cortical areas and layers?

\section{HÖISTAD AND HOF}

There is a plethora of unsuspected pathologic alterations in schizophrenia. Many studies have demonstrated changes in pyramidal neuron populations in various cortical regions (hippocampus, cingulate, dorsolateral prefrontal, primary auditory cortices to name a few). Similarly GABAergic neurons have been shown to be affected, and to present abnormal distribution in frontal and temporal regions alike. Further hinting to a developmental problem is the fact that neurons do not migrate to their appropriate location, or tend to agglomerate in the white matter under the cortex. As such it is not only the ACC that is affected. It may be that some domains of cortex are more vulnerable to an underlying developmental pathology that is revealed postpuberty and may affect different regions in different patients. Assessing the true variability in distribution of changes and their severity among patients with schizophrenia is a daunting task to undertake, but is the necessary neuropathologic index ultimately required to understand the disease process fully, in the context of genetic influences, inferences from animal models and in vitro models, and in vivo functional imaging. This issue speaks to the need of rigorous, extensive, quantitative analyses of postmortem human materials to enhance our understanding of the disease.

\section{A commentary on}

Regulation of myelin genes implicated in psychiatric disorders by functional activity in axons by Lee, P. R., and Fields, R. (2009). Front. Neuroanat. 3:4. doi: 10.3389/neuro.05.004.2009.

\section{REMARKS AND MAIN CONCLUSIONS}

1. The establishment and development of psychiatric disorders are likely to involve aberrant regulation and expression of many genes, together with multiple environmental factors, ultimately leading to illness. In recent years researchers have begun to focus on the potential role of white matter and oligodendrocytes in the pathophysiology of psychiatric disorders.

2. Myelination can be viewed as a highly dynamic process which can be altered by impulse activity in axons and by environmental factors. It is becoming clear that myelination continues into 
adulthood and may contribute to plasticity of cognitive function, learning and memory. Perturbations in the molecular processes leading to axon myelination will consequently result in axon dysfunction and abnormal electrical conduction, therefore impairing the transfer of information across brain regions.

3. There are several mechanisms by which oligodendrocytes could sense functional activity in axons. Oligodendrocytes at various stages of development have ion channels, purinergic and other membrane receptors that allow myelinating glia to detect impulse activity through the activity-dependent release of molecules from axons. Thus activity-dependent regulation of oligodendrocytes could contribute to cellular mechanisms promoting recovery through environmental interventions and other non-drug treatments of psychiatric illnesses.

4. Drug treatments for neuropsychiatric illnesses may also act in part through effects on myelinating glia. Oligodendrocytes have neurotransmitter receptors for glutamate, serotonin, and dopamine, making it likely that antipsychotic drugs acting through these neurotransmitter systems would also have actions on myelinating glia that may be detrimental or beneficial in psychiatric disorders. Finally, synaptic communication between axons and immature myelinating glia (oligodendrocyte progenitor cells), have been described recently in white matter, providing a rapid means of direct communication between axons and myelinating glia.

5. Myelination is a complex biological process that involves an intricate regulatory network among many different cell types in the nervous system. Many of the genes revealed in genomic studies of mental illness that are crucial to the normal functioning of the myelination program and myelin-maintenance are themselves candidates for regulation by electrical activity in axons. Many of these genes relate to oligodendroglia function; however some of these genes are expressed in astrocytes and some in neurons where they may have independent effects or act indirectly on myelinating glia.

6. Regulating transcription of structural components of myelin, such as PLP1, MBP, MAG, MOG, and CNP is clearly critical in the process of oligodendrocyte development and the subsequent correct myelination of specific axons. Several of these major components of myelin have been shown to be regulated by action potential firing or by alterations in intracellular calcium or cAMP; both of these second messengers can be regulated by neural impulse activity.

7. Several of the major myelin genes can be regulated by electrical activity or mechanisms by which second messenger signal transduction can be modified. Many of the major components of myelin are deregulated in psychiatric disorders, particularly in schizophrenia.

8. In order for myelination to proceed, a complex network of transcriptional repression and activation must be activated. Several of the transcription factors required for repression and activation of myelin genes have been found to be abnormally expressed in the brains of patients with psychiatric disorders and in particular those with schizophrenia.

9. Of particular interest is the transcription factor SOX10 which is required for the expression of two of the major components of myelin, the proteins MBP and PLP1. Recently it has been shown that in Schwann cells, Sox10 is a component of a calcium-sensitive transcriptional complex. Calcium is the primary second messenger communicating action potential firing to intracellular responses, and signaling to the nucleus to regulate gene transcription. The Sox10/NFAT complex is critical for Schwann cell development and activates several genes known to be regulators of myelination in the peripheral nervous system. Interestingly the SOX10 gene is located in a major susceptibility locus for schizophrenia and reduced expression of this gene was found to be correlated with an increase in the methylation state of the allele found in schizophrenia patients.

10. External signals generated by electrical activity in axons, such as ATP or glutamate release from axons, can cause changes in intracellular calcium levels in oligodendrocytes and therefore these axonally derived signals may play a role in the epigenetic regulation of the transcriptional apparatus required for lineage progression and myelination by oligodendrocytes and in the process of remyelination. Clearly a perturbation of this type of epigenetic regulation in psychiatric disorders, perhaps by soluble axon-derived signals such as ATP or glutamate, would provide a link with environmental cues as axon firing patterns reflect environmental stimuli.

11. The complexity of mRNA expression and metabolism and the localization of specific mRNAs to subcellular compartments in oligodendrocytes will all contribute to the eventual pool of mRNA available for the translational machinery. RNA transport, splicing and stability mechanisms are tightly regulated by intracellular signal transduction in many cell types in the nervous system. Interestingly, many of the myelin genes are alternatively spliced during development and several of these mRNAs are targets for transport by specific RNA binding proteins. Importantly, a broad spectrum of RNA binding proteins has been found to be deregulated in schizophrenia, including quaking and hnRNAPA2. Deregulation of such a large group of RNA binding proteins will have many downstream consequences for RNA metabolism and localization in oligodendrocytes.

12. Another potential mechanism by which post-transcriptional regulation of oligodendrocyte-related genes may be accomplished is to regulate mRNA homeostasis by the binding of specific micro RNAs (miRNAs) to myelin gene mRNAs. Micro RNAs are regulators of translation and RNA stability; this is achieved by miRNA binding to the UTR of target RNAs and directly influencing the amount of mRNA available to the translational machinery. The study of the regulation of miRNAs in the brain is still at a relatively early stage, however the functional targets of several miRNAs have been described in neurons and oligodendrocytes.

13. In addition to oligodendrocyte-related genes, other nonneuronal genes such as glial fibrillary acidic protein (GFAP), an intermediate filament protein expressed in astrocytes, have been implicated in psychiatric disorders. Astrocytes can have an important influence on development of oligodendrocytes by secretion of trophic factors and cytokines. GFAP is an integral component of the astrocyte cytoskeleton and altered expression of GFAP can have many effects on astrocyte 
biology. Therefore GFAP dysregulation and by implication astrocytic dysfunction could have profound effects on axon health, neuro-transmission, and neuron-glia signaling, and perhaps most importantly indirectly in the process of myelination.

14. Extracellular factors generated by neuronal activity have been shown to regulate GFAP expression and astrocyte differentiation and this could occur through the regulation of chromatin structure. Astrocytes have also been shown to have a direct role in regulating myelination and oligodendrocyte development. Studies in cell culture have shown that astrocytes promote myelination in response to electrical stimulation of axons via the release of a cytokine Leukemia Inhibitory Factor, in response to ATP released from electrically active axons. Taken together it is becoming clear that astrocyte function, which can be regulated by neuronal activity, can have profound effects on the myelination process and may contribute to disease progression in psychiatric disorders.

15. Another mechanism by which electrical activity could influence gene expression in oligodendrocytes is by the expression of cell surface signaling molecules such as specific cell adhesion receptors in axons. This general type of mechanism has already been shown to regulate myelination in the peripheral nervous system.

16. The expression of specific proteins and protein complexes at sites of axon-glia contact could provide a direct link between axon signaling and the regulation of gene expression in oligodendrocytes. In support of this idea it has been demonstrated that extracellular stimuli can regulate the localization of specific mRNAs in axons, and it has been demonstrated that the expression of cell surface receptors can be modulated by electrical activity in axons. Therefore, taken together, regulation of myelination by an activity-dependent signaling cascade, originating in axons, may allow direct coupling of neuronal activity and oligodendrocyte intracellular signaling.

17. It has been demonstrated that diffusible molecules released from axons firing action potentials can be detected by myelinating glia, with subsequent control of glial development and myelination.

18. Adenosine, derived from ATP released from electrically active axons, acts on immature oligodendrocytes to promote differentiation and myelination. Other molecules released by electrically active axons that could in theory influence myelinating glia include potassium and neurotransmitters.

19. Growth factors, such as BDNF, which are known regulators of oligodendrocyte differentiation, can be secreted or regulated in an activity-dependent manner and by environmental experience, providing another potential general mechanism for activity-dependent regulation of myelination. Interactions between BDNF and serotonin in mood disorders have been reported, and the BDNF gene has been associated with increased risk for a number of neuropsychiatric disorders.

20. Drug treatments for psychiatric disorders that correct deregulation of genes involved in myelination and oligodendrocyte dysfunction are an appealing possibility. In this regard pharmacological regulation of the activity of specific histone deacetylases (HDACs) is an interesting avenue of investigation, although therapeutic intervention of the modulation of HDAC activity in mouse models of demyelination has shown mixed results.

21. It may be important to identify cell-type specific regulators of chromatin structure as it relates to oligodendrocyte function, thus targeting any drug treatments more specifically to limit off-target effects on other cell populations in the brain. Regulation of gene expression by medication beyond transcriptional regulation may provide a more specific mechanism to target myelin genes in oligodendrocytes.

22. The extent to which dopamine levels could lead to mental illness in part through effects on myelinating glia, and whether antipsychotic treatments have therapeutic action in part through effects on oligodendrocytes are two intriguing questions of current investigation.

23. Dopamine also can be toxic to oligodendrocyte progenitors by inducing superoxide generation and lowering glutathione levels. Agonists for dopamine D2 and D3 receptors have been shown to provide significant protection of oligodendrocytes against oxidative injury. On the contrary, haloperidol, a typical antipsychotic drug blocking D2 activity reduces myelin proteins in mice treated for 30 days.

24. Schizophrenia and depression can also involve imbalances in the neurotransmitter serotonin, and several drug treatments act through regulating serotonin levels, for example the serotonin reuptake inhibitor fluoxetine (PROZAC). Serotonin receptors are expressed in Schwann cells, and the human polymavirus, JC virus, which causes multifocal leukoencaphalopathy, binds the 5HT2a serotonin receptor on oligodendrocyte progenitor cells.

25. The serotonin antagonists, metoclopramidine, chlorpromazine, clozapine, and serotonin itself all significantly inhibit viral infection, which indicates that medications affecting serotonin levels could influence oligodendrocytes. Indeed, the antidepressant drug, fluoxetine, increases cell proliferation of precursors in cell culture that can give rise to astrocytes, neurons, or oligodendrocytes.

26. Other neurotransmitters can regulate different steps of oligodendrogliogenesis through such ion channels and receptors as the delayed potassium rectifier, the AMPA/kainate, dopamine or muscarinic receptors. This suggests the possibility for activity-dependent regulation of oligodendrocyte differentiation and myelination, and raises the possibility of medications acting on neurotransmitters or the excitation of specific circuits could influence oligodendrocytes.

\section{GENERAL COMMENTS AND DISCUSSION ROCKLAND}

This article presents the myelin environment as an attractive paradigm for investigating coupling of neuronal activity and intracellular signaling, in this case by oligodendrocytes. Another application, emphasized by this and several of the other articles as well, is in the psychiatric domain. Because of its distinctiveness 
and quasi-isolation, white matter may offer some advantages over the more usual gray matter assays in investigating effects, possibly leading to new modes of treatment.

\section{HÖISTAD AND HOF}

Lee and Fields review data indicating that myelination can be altered by activity in axons. Their paper illustrates the global interplay and communication that exists between axons, oligodendrocytes, and astrocytes. Three important aspects are discussed: how activity in axons is sensed by oligodendrocytes and astrocytes, the regulation of myelin and myelin-associated genes, and the relevance for psychiatric disorders.

The activity in axons needs to be sensed by the surrounding oligodendrocytes and astrocytes. For example, this can be done through axon-derived diffusible signals including ATP, adenosine, $\mathrm{K}^{+}$, glutamate, and GABA (Lee and Fields, 2009 Figure 1). [The issue of "diffusing signals" adheres to the concept of volume transmission, originally presented by Fuxe and Agnati (see Agnati et al., 2000; Fuxe et al., 2007) and discussed by Fields (2004)]. It has been shown that oligodendrocytes and astrocytes have receptors and ion channels that enable them to sense the activity in their surrounding. For example, oligodendrocytes have receptors for glutamate, serotonin, and dopamine, as well as purinergic receptors (Fields and Burnstock, 2006). Astrocytes have also been shown to have transmitter receptors (see, e.g., Magistretti et al., 1983; Hosli and Hosli, 1993; Porter and McCarthy, 1997). In addition, synaptic contacts between immature oligodendrocytes and axons have been found, as reviewed by Lee and Fields (Kukley et al., 2007; Karadottir et al., 2008). Astrocytes may also influence oligodendrocyte development by secretion of trophic factors and cytokines. For instance, extracellular factors generated by neuronal activity can regulate GFAP expression in astrocytes. It is possible that GFAP in astrocytes indirectly affects oligodendrocytes and myelination, axons, and neuron-glia interactions. In axons, cell adhesion molecules such as NCAM may influence axon-glia interactions. For example, myelin deposition is targeted to the correct axon site in response to a cell surface receptor expression induced by activity in the axon.

\section{REFERENCES}

Aberg, K., Saetre P., Jareborg, N., and Jazin, E. (2006). Human QKI, a potential regulator of mRNA expression of human oligodendrocyte-related genes involved in schizophrenia. Proc. Natl. Acad. Sci. U.S.A. 103, 7482-7287.

Agnati, L. F., Fuxe, K., Nicholson, C., and Sykova, E. (eds) (2000). Volume transmission revisited. Prog. Brain Res. 125.

Ayoub, A. E., and Kostovic, I. (2009). New horizons for the subplate zone and its pioneering neurons. Cereb. Cortex 19 , 1705-1707.

Cauli, B., Tong, X. K., Rancillac, A., Serluca, N., Lambolez, B., Rossier, J., and Hamel, E. (2004). Cortical GABA interneurons in neurovascular coupling: relays for subcortical vasoactive pathways. J. Neurosci. 24, 8940-8949.

Descarries, L., and Mechawar, N. (2000). Ultrastructural evidence for diffuse transmission by monoamine and acetylcholine neurons of the central nervous system. Prog. Brain Res. 125, 27-47. Interstitial white matter neuron density in the dorsolateral prefrontal cortex and parahippocampal gyrus in schizophrenia. Schizophr. Res. 79, 181-188.

Fan, J., Hof, P. R., Guise, K., Fossella, J. A., and Posner,M.I.(2008). The functional integration of the anterior cingulate cortex during conflict processing. Cereb. Cortex 18, 796-805.

Fields, R. D. (2004). Volume transmission in activity-dependent regulation
Eastwood, S. L., and Harrison, P. J. (2005).

The regulation of myelin or myelin-associated genes can be made either in oligodendrocytes themselves, or via astrocytes and axons. For example, in oligodendrocytes, an important RNA-binding protein, QKI, has been shown to be downregulated in schizophrenia (Katsel et al., 2005; Aberg et al., 2006), which may have downstream consequences on oligodendrocyte development and myelination. It has also been found that the level of QKI mRNA can in fact be influenced by medication used to treat schizophrenia (Aberg et al., 2006). As several antipsychotic medications have their effects through dopamine (as well as serotonin) receptors, Lee and Fields discuss the evidence that dopamine can influence oligodendrocyte development. Although the authors state that "it is unclear exactly how dopamine function is disrupted by oligodendrocyte dysfunction", it should be kept in mind that the axons of the mesolimbic and mesocortical monoamine neurons, which have been classically implicated in the pathophysiology of schizophrenia, are mainly unmyelinated (as observed by Fuxe, 1965a,b; Descarries and Mechawar, 2000). Hence, any potential effects that oligodendrocyte and myelin dysfunction would have on monoamine axons are likely indirect, or rather affect other axons in the circuitry of psychiatric disorders.

\section{FIELDS}

Hoistad and Hof raise an interesting question of what is the pathophysiology of schizophrenia. The literature suggests that the underlying cause of this disorder may be less certain and more complex than the comment suggests. In addition to dopamine, serotonin, and norepinephrine, other neurotransmitter systems, including glutamate, D-serine, and GABA are involved. Anatomical evidence also implicates involvement beyond the mesolimbic and mesocortical pathways. White matter differences have been detected in patients with schizophrenia in widespread brain regions, including prefrontal, hippocampal, temporal, uncinate fasciculus, fornix, cingulate fasciculus, anterior cingulum, superior cerebellar peduncle, and caudate (Fields, 2008, supplemental table). Most would agree that schizophrenia is a complex disorder and it is likely a group of disorders rather than a single disease.

of myelinating glia. Neurochem. Int. $45,503-509$.

Fields, R. D. (2008). White matter in learning, cognition and psychiatric disorders. Trends Neurosci. 31, 361-370.

Fields, R. D., and Burnstock, G. (2006). Purinergic signalling in neuron-glia interactions. Nat. Rev. Neurosci. 7, 423-436.

Fuxe, K. (1965a). Evidence for existence of monoamine neurons in the central nervous system. III. The monoamine nerve terminal. Z. Zellforsch. Mikrosk. Anat. 65, 573-596.

Fuxe, K. (1965b). Evidence for existence of monoamine neurons in the central nervous system. IV. Distribution of monoamine nerve terminals in the central nervous system. Acta Physiol. Scand. Suppl. 247, 37.
Fuxe, K., Dahlström, A., Höistad, M., Marcellino, D., Jansson, A., Rivera, A., Diaz-Cabiale,Z., Jacobsen, K., TinnerStaines, B., Hagman, B., Leo, G., Staines, W., Guidolin, D., Kehr, J., Genedani, S., Belluardo, N., and Agnati, L. F. (2007). From the Golgi-Cajal mapping to the transmitter-based characterization of the neuronal networks leading to two modes of brain communication: wiring and volume transmission. Brain Res. Rev. 55, 17-54.

Hof, P. R., Glezer, I. I., Condé, F., Flagg, R. A., Rubin, M. B., Nimchinsky, E. A., and Vogt Weisenhorn, D. M. (1999). Cellular distribution of the calciumbinding proteins parvalbumin, calbindin, and calretinin in the neocortex of mammals: phylogenetic and developmental patterns. J. Chem. Neuroanat. $16,77-116$. 
Hosli, E., and Hosli, L. (1993). Receptors for neurotransmittors on astrocytes in the mammalian central nervous system. Prog. Neurobiol. 40, 477-506.

Innocenti, G. M., Lehmann, P., and Houzel, J. C. (1994). Computational structure of visual callosal axons. Eur. J. Neurosci. 6, 918-935.

Karadottir, R., Hamilton, N. B., Bakiri, Y., and Atwell, D. (2008). Spiking and nonspiking classes of oligodendrocyte precursor glia in CNS white matter. Nat. Neurosci. 11, 450-456.

Katsel, P., Davis, K. L., and Haroutunian, V. (2005). Variations in myelin and oligodendrocyte-related gene expression across multiple brain regions in schizophrenia: a gene ontology study. Schizophr. Res. 79, 157-173.

Kostovic, I., and Judas, M. (2006). Prolonged coexistence of transient and permanent circuitry elements in the developing cerebral cortex of fetuses and preterm infants. Dev. Med. Child Neurol. 48, 388-393.
Kostovic, I., and Rakic, P. (1990). Developmental history of the transient subplate zone in the visual and somatosensory cortex of the macaque monkey and human brain. J. Comp. Neurol. 297, 441-470.

Kukley, M., Capetillo-Zarate, E., and Dietrich, D. (2007). Vesicular glutamate release from axons in the white matter. Nat. Neurosci. 10, 311-320.

Lee, P. R., and Fields, R. (2009). Regulation of myelin genes implicated in psychiatric disorders by functional activity in axons. Front. Neuroanat. 3:4. doi:10.3389/neuro.05.004.2009.

Magistretti, P. J., Manthorpe, M., Bloom, F. E., and Varon, S. (1983). Functional receptors for vasoactive intestinal polypeptide in cultured astroglia from neonatal rat brain. Regul. Pept. 6, 71-80.

Perrin, J. S., Herve, P. Y., Leonard, G. Perron, M., Pike, G. B., Pitiot, A., Richer, L., Veillette, S., Pausova, Z., and Paus, T. (2008). Growth of white matter in the adolescent brain: role of testosterone and androgen receptor. $J$. Neurosci. 28, 9519-9524.

Porter, J. T., and McCarthy, K. D. (1997). Astrocytic neurotransmitter receptors in situ and in vivo. Prog. Neurobiol. 51, 439-455.

Rinaldi, T., Perrodin, C., and Markram, H. (2008). Hyper-connectivity and hyper-plasticity in the medial prefrontal cortex in the valproic acid animal model of autism. Front. Neural Circuits 2:4. doi: 10.3389/neuro. 04.004.2008.

Rockland, K. S., and Drash, G.W. (1996) Collateralized divergent feedback connections that target multiple areas. J. Comp. Neurol. 373, 529-548.

Segal, D. (2008). Cingulum Bundle Pathophysiology in Schizophrenia. Ph.D. thesis, Department of Neuroscience, Mount Sinai School of Medicine, New York.

Uranova, N., Orlovskaya, D., Vikhreva, O. Zimina, I., Kolomeets, N., Vostrikov, V., and Rachmanova, V. (2001). Electron microscopy of oligodendroglia in severe mental illness. Brain Res. Bull. 55, 597-610.

Yin, X., Crawford, T. O., Griffin, J. W., Tu, P., Lee, V. M., Li, C., Roder, J., and Trapp, B. D. (1998). Myelin associated glycoprotein is a myelin signal that modulates the caliber of myelinated axons. J. Neurosci. 18, 1953-1962.

Received: 13 January 2010; published online: 26 March 2010.

Citation: Front. Neuroanat. (2010) 4:4. doi: 10.3389/neuro.05.004.2010

Copyright (c) 2010 DeFelipe, Fields, Hof, Höistad, Kostovic, Meyer and Rockland. This is an open-access article subject to an exclusive license agreement between the authors and the Frontiers Research Foundation, which permits unrestricted use, distribution, and reproduction in any medium, provided the original authors and source are credited. 